$8-2018$

\title{
Diffusing wild type and sterile mosquitoes in an optimal control setting
}

\author{
K. Renee Fister \\ Murray State University, kfister@murraystate.edu \\ Maeve L. McCarthy \\ mmccarthy@murraystate.edu \\ Seth F. Oppenheimer \\ Mississippi State University
}

Follow this and additional works at: https://digitalcommons.murraystate.edu/faculty

Part of the Other Physical Sciences and Mathematics Commons

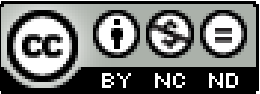

This work is licensed under a Creative Commons Attribution-Noncommercial-No Derivative Works 4.0 License.

\section{Recommended Citation}

This is a preprint manuscript of an article published by Elsevier. The Version of Record of this manuscript has been published and is available in Mathematical Biosciences (ISSN: 0025-5564), May 31, 2018. https://doi.org/10.1016/j.mbs.2018.05.015

This Peer Reviewed/Refereed Publication is brought to you for free and open access by Murray State's Digital Commons. It has been accepted for inclusion in Faculty \& Staff Research and Creative Activity by an authorized administrator of Murray State's Digital Commons. For more information, 


\title{
Diffusing Wild Type and Sterile Mosquitoes in an Optimal Control Setting
}

\author{
K. Renee Fister ${ }^{\mathrm{a}}$, Maeve L. McCarthy ${ }^{1, *}$, Seth F. Oppenheimer ${ }^{\mathrm{b}}$ \\ ${ }^{a}$ Department of Mathematics \& Statistics, Murray State University, Murray KY 42071 \\ ${ }^{b}$ Department of Mathematics \& Statistics, Mississippi State University
}

\begin{abstract}
This paper develops an optimal control framework to investigate the introduction of sterile type mosquitoes to reduce the overal moquito population. As is well known, mosquitoes are vectors of disease. For instance the WHO lists, among other diseases, Malaria, Dengue Fever, Rift Valley Fever, Yellow Fever, Chikungunya Fever and Zika. [http://www.who.int/mediacentre/factsheets/fs387/en/] The goal is to establish the existence of a solution given an optimal sterilization protocol as well as to develop the corresponding optimal control representation to minimize the infiltrating mosquito population while minimizing fecundity and the number of sterile type mosquitoes introduced into the environment per unit time. This paper incorporates the diffusion of the mosquitoes into the controlled model and presents a number of numerical simulations.
\end{abstract}

Key words. Partial differential equations, Optimal control, sterile mosquitoes.

AMS subject classifications (2000). 35L45, 35L50, 92D30.

\section{Introduction}

This paper develops a model for the controlled release of sterile insects into an environment where there is an existing population of wild insects. We will do this under the assumption that the fitness and diffusion rates of the two populations are the same. We will also consider the effect of controlling fecundity by altering the environment in such a way that breeding rate is reduced. This could be done by reducing the locations for breeding though removing sources of standing water and of using larvicide or ovacide. We will not model the use of broad spectrum insecticides because these would also kill our sterilized insects. There has been success in using traps for male insects along with sterile insect release [20], however, we will not consider this third control method in this paper. We note that this work is a generalization of our previous work [14] which did not consider dispersion and spatial variation. Our language in this section follows that of our earlier paper closely.

The importance of controlling mosquito populations is hard to overstate. It is well known that such diseases as yellow fever, dengue fever, epidemic polyarthritis, Rift Valley fever, Ross River Fever, St. Louis encephalitis, West Nile virus, Japanese encephalitis, LaCross encephalitis, Zika, and malaria are carried and transmitted by mosquitoes, [15, 30, 34, 35, 38, 45, 47, 48].

This paper considers a model that can applied to many insects, including mosquitoes. Optimal control theory is then applied with a variety of cost functionals to find the best strategy for reducing insect population at minimal cost.

The sterile insect technique was introduced by Knipling $[19,20]$. The insects are sterilized by irradiation or the application of chemical agents and released to mate with the wild insects. It was used successfully for the screw worm in the late 1950s and early 1960s and great hope was held for using the technique for the control of mosquito populations [21]. Unfortunately, experiments that were carried out with mosquitoes during the same period met with less success. For a discussion of the experimental work in this area see $[5,11,32,44]$.

A number of authors have developed mathematical models of the interaction between sterile and wild mosquitoes, [4, 19, 24, 37]. Recently, models using differences equations, have been developed [27]. Other

\footnotetext{
${ }^{*}$ Corresponding author

Email address: mmccarthy@murraystate.edu (Maeve L. McCarthy)
} 
work considers, as we do, Holling-II type response in population dynamics [6]. Some sterile release models have been explicitly connected to particular diseases $[8,9,46]$. Dumont and Tchuenche [8] consider pulsed sterile release and demonstrate through equilibrium analysis and simulations that frequent small bursts of sterile insects are more effective than larger less frequent releases. An approach by Thome et al. developed in [46] attempts to apply optimal control to both breeding rates and the rate of introduction of sterile mosquitoes. No bounds have been imposed on the control(s) in any of this work which may be not be realistic biologically.

The use of transgenic insects was developed after the sterile insect technique. Insects carrying a dominant lethal gene are introduced into the population. Alphey et al. [2,3] provide many details of the use of both of these techniques. Models that described the interactions of wild and transgenic mosquitoes include those by Li [25, 26] and Diaz et al. [7]. Optimal control methods are applied to the rate of introduction of transgenic mosquitoes by Rafikov et al. [39, 40]. We will not use transgenic mosquitoes in our model.

For a different partial differential equations model for the dynamics of sterile insect release see Alford [1].

It is our hope that by developing new bounded control models for this technique, we may find strategies that will make it more effective.

\subsection{The model}

We are particularly interested in Li's model of the release of transgenic mosquito populations [26] which we modified in our earlier paper to model the release of sterile mosquitoes. Although our focus is on sterile mosquitoes, we will follow the approach in the referenced paper for the model we develop here because it captures the features we seek to incorporate.We start by concentrating on the local dynamics. We consider a population of wild mosquitoes, $W$, and a population of sterilized mosquitoes, $S$. If $R(W, S)$ is the birth rate of the wild mosquitoes and $d_{W}(W, S)$ and $d_{S}(W, S)$ are the death rates of the the wild population and sterilized population respectively, we obtain

$$
\begin{aligned}
\frac{d W}{d t} & =W\left(R(W, S)-d_{W}(W, S)\right) \\
\frac{d S}{d t} & =-S d_{S}(W, S)+B(t)
\end{aligned}
$$

where $B$ is the release rate of sterile mosquitoes. We will assume the death rate has a constant component and a component that increases with total population density. Thus we will have

$$
\begin{aligned}
d_{W}(W, S) & =M+K(W+S) \\
d_{S}(W, S) & =M+K(W+S)
\end{aligned}
$$

where the equality of the constants is an implicit assumption of equal fitness between the wild population and the sterilized population. We now turn our attention to the birthrate, $R(W, S)$.

Continuing to follow the approach in [26] and our earlier paper [14], we let $c(W, S, t)$ be the number of matings that occur per unit time. Therefore, we can expect that the number of matings of wild type to wild type will be

$$
R(W, S)=c(W, S, t) \frac{W}{W+S}
$$

This will give us

$$
\begin{aligned}
\frac{d W}{d t} & =W\left(c(W, S, t) \frac{W}{W+S}-M-K(W+S)\right) \\
\frac{d S}{d t} & =-S(M+K(W+S))+B(t)
\end{aligned}
$$

Let us consider a couple of choices for the function $c(W, S, t)$. When the total population is large, we expect that mosquitoes will have no difficulty finding a mate, giving us $c(W, S, t)$ as a function only of time, $A(t)$ which is the product of such factors as the likelihood of a mating producing eggs, the (fixed) proportion of the population that is female, the likelihood that an appropriate place can be found so that when the eggs are laid they will hatch, and so on. $A(t)$ can be reduced through the application of larvicide or 
insecticide, the clearing of breeding sites, etc. Henceforth, we will generally refer to such habitat modification as the application of larvicide, with the understanding that habitat modification can have other features. The function $A(t)$ will serve as a control as well as $B$, since we are assuming we can take action to reduce the amount of suitable real estate for successful egg laying. This gives the following model

$$
\begin{aligned}
\frac{d W}{d t} & =W\left(\frac{A(t) W}{W+S}-M-K(W+S)\right) \\
\frac{d S}{d t} & =-S(M+K(W+S))+B(t)
\end{aligned}
$$

When the population is relatively small, we expect the law of mass action to be pertinent with $c(W, S, t)=$ $A(t)(W+S)$ where the function $A(t)$ is similar to the function $A(t)$ described above. This gives us

$$
\begin{aligned}
\frac{d W}{d t} & =W(A(t) W-M-K(W+S)) \\
\frac{d S}{d t} & =-S(M+K(W+S))+B(t)
\end{aligned}
$$

We are particularly interested in a function that can capture the dynamics of both large and small populations simultaneously. We seek a functional form that will lead to approximately the models above. Once again, we follow the work of Li [26] and our previous work [14], and choose a Holling-II-type functional response, [17]. Fixing a positive constant $\varepsilon>0$, we set

$$
c(W, S)=A \frac{W+S}{\varepsilon+W+S}
$$

giving us

$$
\begin{aligned}
\frac{d W}{d t} & =W\left(\frac{A W}{\varepsilon+W+S}-M-K(W+S)\right) \\
\frac{d S}{d t} & =-S(M+K(W+S))+B(t)
\end{aligned}
$$

We are now ready to add in diffusion terms under the assumption that both the wild type and sterile mosquitoes diffuse with constant $D$. Henceforth, we assume all functions are functions of space and time.

$$
\begin{aligned}
\frac{\partial W}{\partial t} & =D \triangle W+W\left(\frac{A W}{\varepsilon+W+S}-M-K(W+S)\right) \\
\frac{\partial S}{\partial t} & =D \triangle S-S(M+K(W+S))+B(x, t)
\end{aligned}
$$

We now rescale in order to make the mathematical analysis more tractable, letting $w=\frac{W}{\varepsilon}$ and $s=\frac{S}{\varepsilon}$.

$$
\begin{aligned}
\varepsilon \frac{\partial w}{\partial t} & =\varepsilon D \Delta w+\varepsilon w\left(\frac{A w \varepsilon}{\varepsilon+\varepsilon w+\varepsilon s}-M-K \varepsilon(w+s)\right) \\
\varepsilon \frac{\partial s}{\partial t} & =\varepsilon D \Delta s-\varepsilon s(M+K \varepsilon(w+s))+B(x, t)
\end{aligned}
$$

Setting $\kappa=D, \alpha=A, \mu=M \varepsilon, \eta=K \varepsilon, \beta(x, t)=\frac{B(x, t)}{\varepsilon}$ yields our final model,

$$
\begin{aligned}
\frac{\partial w}{\partial t} & =\kappa \Delta w+\varepsilon\left(\frac{\alpha w}{1+w+s}-\mu-\eta(w+s)\right) \\
\frac{\partial s}{\partial t} & =\kappa \Delta s-s(\mu+\eta(w+s))+\beta(t) .
\end{aligned}
$$

where the initial conditions are

$$
w(x, 0)=w_{0}, s(0)=s_{0}
$$


and the controls are bounded with $M_{1}, M_{2}, N_{1}, N_{2} \geq 0$ such that

$$
M_{1} \leq \alpha(t) \leq M_{2}, \quad N_{1} \leq \beta(t) \leq N_{2}
$$

The rest of this paper is organized as follows. In section 2, we establish existence results for the PDE model. In section 3, we obtain the existence of an optimal control pair $(\alpha, \beta)$ for our objective functionals. In section 4 , we derive optimality conditions that characterize the optimal control pair. In section 5 we implement numerical simulations using parameters for Anopheles gambiae. Finally, in section 6, we provide discussion of our results and their implications for the optimal control of mosquito populations.

\section{Discussion of Model}

We investigate the dimensionless model with $w(x, t)$ and $s(x, t)$ representing the density of the wild type mosquitoes and the sterile type, respectively. The mosquitoes are governed by a reaction-diffusion equation system in the domain $Q=\Omega \times(0, T)$ with $x \in \Omega$ and finite $\mathrm{T}$ as follows:

$$
\begin{aligned}
\frac{\partial w}{\partial t} & =\kappa \Delta w+w\left[\frac{\alpha w}{1+w+s}-(\mu+\eta(w+s))\right] \\
\frac{\partial s}{\partial t} & =\kappa \Delta s-s(\mu+\eta(w+s))+\beta(x, t) \\
\left(\begin{array}{c}
w(x, 0) \\
s(x, 0)
\end{array}\right) & =\left(\begin{array}{c}
w_{0}(x) \\
s_{0}(x)
\end{array}\right) \text { for } x \in \Omega \\
\left(\begin{array}{c}
\nabla w(x, t) \cdot \nu \\
\nabla s(x, t) \cdot \nu
\end{array}\right) & =-\left(\begin{array}{c}
w(x, t) \\
s(x, t)
\end{array}\right) \text { for } x \in \partial \Omega \text { and } t>0
\end{aligned}
$$

Here $\nu$ is the unit outward normal on $\partial \Omega$ and we are using homogeneous Robin conditions.

We first develop continuity and ellipticity conditions for our bilinear form and then prove that this system has a unique positive bounded solution. We have our solution set such that $(w, s) \in W \times W$ where $W=$ $L^{2}\left((0, T) ; H^{1}(\Omega)\right)$.

\subsection{Existence of Solutions}

We start by considering the linear operator on $\left(L^{2}(\Omega)\right)^{2}$.

$$
\begin{aligned}
L\left(\begin{array}{c}
w(x) \\
s(x)
\end{array}\right) & =\left(\begin{array}{c}
\kappa \Delta w-\mu w \\
\kappa \Delta s-\mu s
\end{array}\right) \\
\left(\begin{array}{c}
\nabla w(x) \cdot \nu \\
\nabla s(x) \cdot \nu
\end{array}\right) & =-\left(\begin{array}{c}
w(x) \\
s(x)
\end{array}\right) \text { for } x \in \partial \Omega
\end{aligned}
$$

Notice that this is diagonal. If we can show that one coordinate generates a analytic semigroup on $L^{2}(\Omega)$ then the diagonal operator will generate an analytic semigroup on the product space. We use the following from Showalter's book [42].

Theorem 2.1. Let $V$ and $H$ be Hilbert spaces for which the identity $H \hookrightarrow V$ is continuous. Let $a: V \times V \rightarrow$ $\mathrm{C}$ be sesquilinear and satisfy there are two constants $0<c \leq K$ so that

1. $|a(w, v)| \leq\|w\|_{V}\|v\|_{V}$ for all $w$ and $v$ in $V$ (continuity)

2. Re $a(v, v) \geq c\|v\|_{V}^{2}$ for all $v$ in $V$ ( $V$ elliptic $)$.

Proof. Define

$$
D(A)\left\{w \in V:|a(w, v)| \leq K_{w}|v|_{H}, v \in V\right\}
$$

where $K_{w}$ depends only on $w$ and let $A$, a linear function from $D(A)$ to $H$, be given by

$$
(A w, v)_{H}=a(w, v), w \in D(A), v \in V
$$


Then $D(A)$ is dense in $H$ and $-A$ generates an analytic semigroup on $H$.

We will define $H=L^{2}(\Omega)$ and $V=W^{1,2}(\Omega)$. Our form is

$$
a(w, \phi)=\int_{\Omega} \kappa \nabla w \cdot \nabla \phi+\mu w \phi d x+\int_{\partial \Omega} w \phi d x
$$

We start with continuity. We use the trace theorem that $W^{1,2}(\Omega)$ embeds continuously, hence boundedly, into $L^{2}(\partial \Omega)$ with bound we will call $K_{T}$.

$$
|a(w, \phi)| \leq \int_{\Omega} \kappa|\nabla w||\nabla \phi|+\mu|w||\phi| d x+\int_{\partial \Omega}|w||\phi| d x
$$

Applying Hölder's inequality and the fact that for real numbers $a$ and $b, a b \leq \frac{1}{2}\left(a^{2}+b^{2}\right)$ we have the above is bounded by

$$
\kappa\|\nabla w\|_{H}\|\nabla \phi\|_{H}+\mu\|w\|_{H}\|\phi\|_{H}+\|w\|_{L^{2}(\partial \Omega)}\|\phi\|_{L^{2}(\partial \Omega)}
$$

Since each of $\|\nabla w\|_{H},\|w\|_{H}$, and $\|w\|_{L^{2}(\partial \Omega)}$ is bounded by a positive multiple of $\|w\|_{V}$ we have estimate 1 .

To see estimate 2 ,

$$
\begin{aligned}
a(w, w) & =\int_{\Omega} \kappa \nabla w \cdot \nabla w+\mu w^{2} d x+\int_{\partial \Omega} w^{2} d x \\
& \geq \int_{\Omega} \kappa \nabla w \cdot \nabla w+\mu w^{2} d x \\
& \geq \min (\kappa, \mu)\|w\|_{V}
\end{aligned}
$$

and we have the function $-A$ defined in theorem A of Showalter, [42], generates an analytic semigroup on $L^{2}(\Omega)$. Now let's show that this operator $A$ and $D(A)$ are what we want.

Let $\phi$ be any compactly supported infinitely differentiable function on $\Omega$. For an $u$ in $D(A)$ there is a constant $K_{w}$ so that

$$
\begin{aligned}
K_{u}\|\phi\|_{H} & \geq|a(w, \phi)| \\
& =\left|\int_{\Omega} \kappa \nabla w \cdot \nabla \phi+\mu w \phi d x+\int_{\partial \Omega} w \phi d x\right| \\
& =\left|\int_{\Omega}-\kappa \Delta w \phi+\mu w \phi d x\right| \\
& =\left|\int_{\Omega}(-\kappa \Delta w+\mu w) \phi d x\right|
\end{aligned}
$$

where we have used the definition of distributional derivative. Seeing that $-\kappa \Delta w+\mu w$ defines a continuous linear functional on $H$, we get that $-\kappa \Delta w+\mu w \in H$ and thus $\Delta w \in H$. Let $\phi$ be any compactly supported infinitely differentiable function on $\Omega$ and $B$ on $D(A)$ by $B w=-\kappa \Delta w+\mu w$. Then

$$
\begin{aligned}
(A w, \phi)-(B w, \phi) & =a(w, \phi)-(B w, \phi) \\
& =0
\end{aligned}
$$

Since $C_{0}^{\infty}(\Omega)$ is dense in $H$, we have that $A=B$. Finally, let $w \in D(A)$ and let $\phi$ be any infinitely differentiable function on $\Omega$. Then

$$
\begin{aligned}
0 & =a(w, \phi)-(A w, \phi) \\
& =\int_{\Omega} \kappa \nabla w \cdot \nabla \phi+\mu w \phi d x+\int_{\partial \Omega} w \phi d x-\int_{\Omega}-\kappa \Delta w \phi+\mu w \phi d x \\
& =\int_{\Omega} \kappa \nabla w \cdot \nabla \phi+\mu w \phi d x+\int_{\partial \Omega} w \phi d x-\left(\int_{\partial \Omega}-\kappa \frac{\partial w}{\partial \nu} \phi d x+\int_{\Omega} \kappa \nabla w \cdot \nabla \phi+\mu w \phi d x\right) \\
& =\int_{\partial \Omega} w \phi+\kappa \frac{\partial w}{\partial \nu} \phi d x
\end{aligned}
$$


Since $\phi$ was arbitrary and we have

$$
D(A)=\left\{w \in W^{1,2}(\Omega): \Delta w \in L^{2}(\Omega), \kappa \frac{\partial w}{\partial \nu}=-w \text { on } \partial \Omega\right\} .
$$

the diagonal operator $L$ is exactly what we want. We now define

$$
g(w, s)=\left[\begin{array}{c}
w\left[\frac{\alpha w}{1+w+s}-\eta(w+s)\right] \\
-s \eta(w+s)
\end{array}\right]
$$

We fix some large positive number $M$ and define

$$
\underline{\bar{g}}(w, s)=g\left(\min \left(w^{+}, M\right), \min \left(s^{+}, M\right)\right)
$$

This function $\bar{g}(w, s)$ does map $L^{2}(\Omega) \times L^{2}(\Omega)$ into itself and is Lipschitz. Thus, for each $\left(w_{0}, s_{0}\right) \in$ $L^{2}(\Omega) \times L^{2}(\Omega)$, the initial value problem

$$
\begin{aligned}
\frac{\partial}{\partial t}\left[\begin{array}{c}
w \\
s
\end{array}\right] & =L\left[\begin{array}{c}
w \\
s
\end{array}\right]+\bar{g}(w, s) \\
{\left[\begin{array}{c}
w \\
s
\end{array}\right](\cdot, 0) } & =\left[\begin{array}{c}
w_{0} \\
s_{0}
\end{array}\right](\cdot)
\end{aligned}
$$

has a unique mild solution. We may now bootstrap to observe that the solution is continuous

$$
\left[\begin{array}{c}
w \\
s
\end{array}\right](\cdot, t)
$$

in $t$ and thus so is $\underline{g}(w(\cdot, t), s(\cdot, t))$ which implies that

$$
\left[\begin{array}{c}
w \\
s
\end{array}\right](\cdot, t)
$$

is Hölder continuous in $t$, Pazy, chapter 4 theorem 3.1, [36] and thus so is $\underline{g}(w(\cdot, t), s(\cdot, t))$ which implies that

$$
\left[\begin{array}{c}
w \\
s
\end{array}\right](\cdot, t)
$$

is a classical solution by Pazy, chapter four corollary 3.3, [36].

We will now prove upper and lower bounds that show that the original function $g$ may be used with nonnegative essentially bounded $\left(w_{0}, s_{0}\right)$ and yields nonnegative essentially bounded solutions.

We define two functions

$$
\rho_{k}(t)=\left\{\begin{array}{cc}
0 & t \leq 0 \\
k t & t \in\left(0, \frac{1}{k}\right) \\
1 & t \geq \frac{1}{k}
\end{array}\right.
$$

It is easy to see that this Lipschitz function satisfies

$$
\rho_{k}(t) \rightarrow \operatorname{sgn}_{0}^{+}(t) \text { as } k \rightarrow \infty
$$

We define

$$
\sigma_{k}(t)=\int_{0}^{t} \rho_{k}(\tau) d \tau
$$

and we similarly observe that

$$
\sigma_{k}(t) \rightarrow t^{+} \text {as } k \rightarrow \infty
$$

We now observe that

$$
\min \left(w^{+}, M\right) \rho_{k}(-w)=0
$$


Thus

$$
\begin{aligned}
w_{t} \rho_{k}(-w)= & \kappa \Delta w \rho_{k}(-w)-\mu w \rho_{k}(-w) \\
& +\min \left(w^{+}, M\right)\left[\frac{\alpha \min \left(w^{+}, M\right)}{1+\min \left(w^{+}, M\right)+\min \left(s^{+}, M\right)}\right. \\
& \left.-\eta\left(\min \left(w^{+}, M\right)+\min \left(s^{+}, M\right)\right)\right] \rho_{k}(-w) \\
= & \kappa \Delta w \rho_{k}(-w)-w \rho_{k}(-w)
\end{aligned}
$$

Thus

$$
w_{t} \rho_{k}(-w)=\kappa \Delta w \rho_{k}(-w)-\mu w \rho_{k}(-w) .
$$

Integrating over $\Omega$ gives us

$$
\begin{aligned}
-\frac{\partial}{\partial \tau} \int_{\Omega} \sigma_{k}(-w) d x & =\int_{\Omega} \kappa \Delta w \rho_{k}(-w)-\mu w \rho_{k}(-w) d x \\
& \geq \int_{\Omega} \kappa \Delta w \rho_{k}(-w) d x
\end{aligned}
$$

Where we have used $\mu w \rho_{k}(-w) \leq 0$. Thus

$$
\begin{aligned}
-\frac{\partial}{\partial \tau} \int_{\Omega} \sigma_{k}(-w) d x & \geq \int_{\Omega} \kappa \Delta w \rho_{k}(-w) d x \\
& =\int_{\partial \Omega} \kappa \frac{\partial w}{\partial \nu} \rho_{k}(-w) d S+\int_{\Omega} \kappa\|\nabla w\|^{2} \varkappa_{\left\{x \in\left(0, \frac{1}{k}\right)\right\}} d x \\
& \geq \int_{\partial \Omega} \kappa \frac{\partial w}{\partial \nu} \rho_{k}(-w) d S \\
& =\int_{\partial \Omega}-w \rho_{k}(-w) d S \\
& \geq 0
\end{aligned}
$$

Thus

$$
\begin{aligned}
\int_{\Omega} \sigma_{k}(-w(x, t)) d x & \leq \int_{\Omega} \sigma_{k}\left(-w_{0}(x, t)\right) d x \\
& =0 .
\end{aligned}
$$

Moreover,

$$
w(x, t) \geq 0 \text { a.e. } x \text { and all } t \geq 0 .
$$

Note that since since $w(\cdot, t) \in D(A)$ for $t>0$, the inequality will hold everywhere. A similar, but easier, argument gives positivity for $s$.

We now assume that $w$ and $s$ are non negative and define

$$
\bar{g}(w, s)=g(\min (w, M), \min (s, M))
$$

We now obtain boundedness for $s$. We again take our equation for $s$ and multiply by $\rho_{k}(s-R)$ and require that $M>R$. In any practical application there will be a maximum value for $\beta(x, t)$ we let both $R$ and $\mu R$ be larger than this maximum and $\left\|w_{0}\right\|_{\infty}$. We observe that $\rho_{k}(s-R), w$ and $s$ are all non negative and compute

$$
\begin{aligned}
s_{t} \rho_{k}(s-R)= & \kappa \Delta s \rho_{k}(s-R)-\mu s \rho_{k}(s-R)+\beta(x, t) \\
& -\eta\left(\min (s, M)(\min (w, M)+\min (s, M)) \rho_{k}(s-R)\right) \\
\leq & \kappa \Delta s \rho_{k}(s-R)+\beta(x, t) \rho_{k}(s-R)
\end{aligned}
$$


We may now integrate over $\Omega$ to obtain

$$
\begin{aligned}
\frac{\partial}{\partial t} \int_{\Omega} \sigma_{k}(s-R) d x \leq & \int_{\Omega} \kappa \Delta s \rho_{k}(s-R)-\mu s \rho_{k}(s-R) d x+\int_{\Omega} \beta(x, t) \rho_{k}(s-R) d x \\
= & \int_{\partial \Omega} \kappa \frac{\partial s}{\partial \nu} \rho_{k}(s-R) d s-\int_{\Omega} \kappa\|\nabla s\|^{2} \varkappa_{\left\{s \in\left(0, \frac{1}{k}\right)\right\}} d x \\
& -\int_{\Omega} \mu s \rho_{k}(s-R) d x+\int_{\Omega} \beta(x, t) \rho_{k}(s-R) d x \\
\leq & \int_{\partial \Omega}-u \rho_{k}(s-R) d s+\int_{\Omega} \beta(x, t) d x \\
\leq & -\int_{\Omega} \mu s \rho_{k}(s-R) d x+\int_{\Omega} \beta(x, t) \rho_{k}(s-R) d x \\
\leq & -\int_{\Omega} \mu s \rho_{k}(s-R) d x+\int_{\Omega} \mu R \rho_{k}(s-R) d x \\
\leq & -\int_{\Omega} \mu(s-R) \rho_{k}(s-R) d x \\
\leq & 0
\end{aligned}
$$

Hence

$$
\int_{\Omega} \sigma_{k}(s(x, t)-R) d x \leq \int_{\Omega} \sigma_{k}\left(s_{0}(x)-R\right) d x
$$

Letting $k \rightarrow \infty$ and using the Lebesgue dominated convergence theorem yields

$$
\begin{aligned}
\int_{\Omega}(s(x, t)-R)^{+} d x & \leq \int_{\Omega}\left(s_{0}(x)-R\right)^{+} d x \\
& =0
\end{aligned}
$$

and $s \leq R$.

We now do the (easier) case of $w$. We multiply by $\rho_{k}(w-R)$, where

$$
R>\max \left\{\frac{\alpha}{\eta},\left\|w_{0}\right\|_{\infty}\right\}
$$

and require that $M>R$. Note that $(\alpha-\eta s) \rho_{k}(s-R) \leq 0$ for all real $s$ and, since $M>R$,

$$
(\alpha-\eta \min (s, M)) \rho_{k}(s-R) \leq 0 .
$$

Thus

$$
\begin{aligned}
w_{t} \rho_{k}(w-R)= & \kappa \Delta w \rho_{k}(w-R)-\mu w \rho_{k}(w-R) \\
& +\min (w, M)\left[\frac{\alpha \min (w, M)}{1+\min (w, M)+\min (s, M)}\right. \\
& \left.-\eta\left(\min \left(w^{+}, M\right)+\min \left(s^{+}, M\right)\right)\right] \rho_{k}(w-R) \\
\leq & \kappa \Delta w \rho_{k}(w-R)+\min (w, M)\left[\alpha-\eta\left(\min \left(w^{+}, M\right)\right)\right] \rho_{k}(w-R) \\
\leq & \kappa \Delta w \rho_{k}(w-R)
\end{aligned}
$$

We may now integrate over $\Omega$ and obtain

$$
\begin{aligned}
\frac{\partial}{\partial t} \int_{\Omega} \sigma_{k}(w(x, t)-R) d x & \leq \int_{\Omega} \kappa \Delta w \rho_{k}(w-R) \\
& =\int_{\partial \Omega} \kappa \frac{\partial w}{\partial \nu} \rho_{k}(w-R) d s-\int_{\Omega} \kappa\|\nabla w\|^{2} \varkappa_{\left\{w \in\left(0, \frac{1}{k}\right)\right\}} d x \\
& \leq \int_{\partial \Omega}-w \rho_{k}(w-R) d s \\
& \leq 0
\end{aligned}
$$


Thus

$$
\int_{\Omega} \sigma_{k}(w(x, t)-R) d x \leq \int_{\Omega} \sigma_{k}\left(w_{0}(x)-R\right) d x
$$

Letting $k \rightarrow \infty$ and using the Lebesgue dominated convergence theorem yields

$$
\begin{aligned}
\int_{\Omega}(w(x, t)-R)^{+} d x & \leq \int_{\Omega}\left(w_{0}(x)-R\right)^{+} d x \\
& =0
\end{aligned}
$$

and $w \leq R$.

Theorem 2.2. The original system (4-7)

$$
\begin{aligned}
\frac{\partial w}{\partial t} & =\kappa \Delta w+w\left[\frac{\alpha w}{1+w+s}-(\mu+\eta(w+s))\right] \\
\frac{\partial s}{\partial t} & =\kappa \Delta s-s(\mu+\eta(w+s))+\beta(x, t) \\
\left(\begin{array}{c}
w(x, t) \\
s(x, t)
\end{array}\right) & =\left(\begin{array}{c}
w_{0}(x) \\
s_{0}(x)
\end{array}\right) \text { for } x \in \Omega \\
\left(\begin{array}{c}
\nabla w(x, t) \cdot \nu \\
\nabla s(x, t) \cdot \nu
\end{array}\right) & =-\left(\begin{array}{c}
w(x, t) \\
s(x, t)
\end{array}\right) \text { for } x \in \partial \Omega \text { and } t>0
\end{aligned}
$$

has a unique positive classical solution which is bounded in $L^{\infty}(\Omega) \times L^{\infty}(\Omega)$ whenever the initial pair is nonnegative and bounded in $L^{\infty}(\Omega) \times L^{\infty}(\Omega)$.

Since we have the existence for the solution for the mosquito system for all time, we can investigate the optimal control strategy associated with different objective functional subject to the original system (2)-(3).

\section{Existence of Optimal Controls}

In this section and for the remainder of the paper, we use $w_{t}$ instead of $\frac{\partial w}{\partial t}$ for partial derivatives with respect to time.

In the discussion of the existence of an optimal control pair, we consider the variational form of our original system for $w, s, \phi, \psi \in W$ in the following sense with $\Gamma=\partial \Omega \times(0, T)$.

$$
\begin{gathered}
\int_{0}^{T}\left\langle w_{t}, \phi\right\rangle d t+\kappa \int_{0}^{T} \int_{\Omega} \nabla w \nabla \phi d x d t=\int_{0}^{T} \int_{\Omega} w\left[\frac{\alpha w}{1+w+s}-(\mu+\eta(w+s))\right] \phi d x d t-\int_{\Gamma} w \phi d s \\
\text { and } \\
\int_{0}^{T}\left\langle s_{t}, \psi\right\rangle d t+\kappa \int_{0}^{T} \int_{\Omega} \nabla s \nabla \psi d x d t=\int_{0}^{T} \int_{\Omega}\{-s(\mu+\eta(w+s))+\beta(x, t)\} \psi d x d t-\int_{\Gamma} s \psi d s
\end{gathered}
$$

where $\langle$,$\rangle inner product is the duality between \left(H^{1}(\Omega)\right)^{*}$ and $H^{1}(\Omega)$.

\subsection{Objective Functional}

In order to seek an optimal control, we need to define an admissible control set, $\mathcal{A}$.

$\mathcal{A}=\left\{(\alpha, \beta) \in\left(L^{\infty}(Q)\right)^{2} \mid M_{1} \leq \alpha(x, t) \leq M_{2}\right.$ and $N_{1} \leq \beta(x, t) \leq N_{2}$ a.e. in $\left.Q\right\}$. We seek to minimize the objective functional, $J(\alpha, \beta)$ over $\mathcal{A}$ where

$$
J(\alpha, \beta)=\int_{Q}\left\{K_{1} w+K_{2} s+\frac{1}{2}\left(K_{3} \alpha^{2}+K_{4} \beta^{2}\right)\right\} d x d t
$$

so that the density of the wild and transgenic type mosquitoes are minimized along with the nonlinear cost associated with the control of the fecundity term, $\alpha(x, t)$ and the release rate of the sterile mosquitoes, $\beta(x, t)$. 
The different terms have different associated weights $K_{i}$ for $i=1, \ldots 4$ that represent environmental factors and monetary weights of the cost of the control program.

To proceed with the optimal control development, we need to obtain the existence of the optimal control pair that minimizes the aforementioned functional.

Theorem 3.1. There exists and optimal control pair in $\mathcal{A}$ that minimizes the functional $J(\alpha, \beta)$.

Proof. Since the state variables and the controls are bounded below, we have that

$$
\inf _{(\alpha, \beta) \in \mathcal{A}}\{J(\alpha, \beta) \mid(\alpha, \beta) \in \mathcal{A}\}<\infty .
$$

There exists a minimizing sequence $\left(\alpha^{n}, \beta^{n}\right) \in \mathcal{A}$ such that

$$
\lim _{n \rightarrow \infty} J\left(\alpha^{n}, \beta^{n}\right)=\inf _{(\alpha, \beta) \in \mathcal{A}}\{J(\alpha, \beta) \mid(\alpha, \beta) \in \mathcal{A}\}
$$

By the existence and uniqueness of a solution of the state system in Theorem 2.2, we can define

$$
w^{n}=w\left(\alpha^{n}, \beta^{n}\right) \text { and } s^{n}=s\left(\alpha^{n}, \beta^{n}\right)
$$

for each $\mathrm{n}$. Using the weak formulations to the state system as noted in equations (8) - (9) for $w^{n}$ and $s^{n}$, we develop estimates in order to discuss convergence of our sequences. With appropriate test functions in the weak formulations, we obtain

$$
\begin{array}{r}
\int_{0}^{t}\left\langle w_{t}^{n}, w^{n}\right\rangle d t+\kappa \int_{0}^{t} \int_{\Omega}\left|\nabla w^{n}\right|^{2}+\left|\nabla s^{n}\right|^{2} d x d t=\int_{0}^{t} \int_{\Omega} w^{n}\left[\frac{\alpha^{n} w^{n}}{1+w^{n}+s^{n}}-\left(\mu+\eta\left(w^{n}+s^{n}\right)\right)\right] w^{n} d x d t \\
-\int_{\Gamma}\left(w^{n}\right)^{2} d s-\int_{\Gamma}\left(s^{n}\right)^{2} d s+\int_{0}^{t} \int_{\Omega}\left\{-s^{n}\left(\mu+\eta\left(w^{n}+s^{n}\right)\right)+\beta^{n}(x, t)\right\} s^{n} d x d t .
\end{array}
$$

We note that

$$
\int_{0}^{t}\left\langle w_{t}^{n}, u^{n}\right\rangle d t=\frac{1}{2} \int_{\Omega}\left\{\left\{w^{n}(x, t)\right\}^{2}-\left\{w_{0}(x, t)\right\}^{2}\right\} d x
$$

Since $\left(\mu+\eta\left(w^{n}+s^{n}\right)\right)>0, \int_{\Gamma}\left\{\left(w^{n}\right)^{2}+\left(s^{n}\right)^{2}\right\} d s \geq 0$, and that $w^{n}$ solution is bounded, then with using the bounds on the controls, we have

$$
\begin{array}{r}
\frac{1}{2} \int_{\Omega}\left\{\left(w^{n}\right)^{2}+\left(s^{n}\right)^{2}\right\} d x+\kappa \int_{0}^{t} \int_{\Omega}\left|\nabla w^{n}\right|^{2}+\left|\nabla s^{n}\right|^{2} d x d t \\
\leq \frac{1}{2} \int_{\Omega}\left\{w_{0}(x, t)\right\}^{2}+\left\{s_{0}(x, t)\right\}^{2} d x+M_{3} \int_{0}^{t} \int_{\Omega}\left(w^{n}\right)^{2} d x d t+N_{2} \int_{0}^{t} \int_{\Omega} s^{n} d x d t
\end{array}
$$

where $M_{3}$ depends on $N=M_{2}$ and the bound of $w^{n}$. Next, we use Cauchy's inequality on $N_{2} \int_{0}^{t} \int_{\Omega} s^{n} d x d t$ so that we can bound this term by a term involving the square of $s^{n}$ and a constant $N_{3}$ depending on $N_{2}$ and the bound of the domain. Using the aforementioned Cauchy estimate, we see that the first integral term in the estimate (12) is bounded as

$$
\int_{\Omega}\left\{\left(w^{n}\right)^{2}+\left(s^{n}\right)^{2}\right\} d x \leq N_{3}+\int_{\Omega}\left\{w_{0}(x, t)\right\}^{2}+\left\{s_{0}(x, t)\right\}^{2} d x+M_{4} \int_{0}^{t} \int_{\Omega}\left\{\left(w^{n}\right)^{2}+\left(s^{n}\right)^{2}\right\} d x d t
$$

where $M_{4}=2 M_{3}+1$. We apply Gronwall's Theorem to the inequality (13) and receive

$$
\int_{\Omega}\left\{\left(w^{n}\right)^{2}+\left(s^{n}\right)^{2}\right\} d x \leq e^{M_{4} T}\left\{N_{3}+\int_{\Omega}\left\{w_{0}(x, t)\right\}^{2}+\left\{s_{0}(x, t)\right\}^{2} d x .\right\}
$$


Using the Gronwall estimate and the estimate in (13), we see that the estimate in (12) becomes

$$
\begin{array}{r}
\sup _{0 \leq t \leq T}\left\{\int_{\Omega}\left[\left(w^{n}\right)^{2}+\left(s^{n}\right)^{2}\right] d x+2 \kappa \int_{0}^{t} \int_{\Omega}\left|\nabla w^{n}\right|^{2}+\left|\nabla s^{n}\right|^{2} d x d t\right\} \\
\leq \int_{\Omega}\left[\left\{w_{0}(x, t)\right\}^{2}+\left\{s_{0}(x, t)\right\}^{2}\right] d x+N_{3}+M_{4} \int_{0}^{t} \int_{\Omega}\left\{\left(w^{n}\right)^{2}+\left(s^{n}\right)^{2}\right\} d x d t \\
\leq C_{1} e^{M_{4} T}\left\{N_{3}+\int_{\Omega}\left\{w_{0}(x, t)\right\}^{2}+\left\{s_{0}(x, t)\right\}^{2} d x\right.
\end{array}
$$

where $C_{1}$ depends on $M_{4}$ and $T$.

In order to obtain our result, we need to pass to the limit in the $\left(w^{n}, s^{n}\right)$ partial differential equation system. From the estimates and embedding theory, we find the necessary convergences. Specifically, we can extract subsequences since $w^{n}$ and $s^{n}$ are bounded in $W$ such that $w^{n} \rightarrow w^{*}$ and $s^{n} \rightarrow s^{*}$ in $W$. Additionally, we see that $w_{t}^{n}$ and $s_{t}^{n}$ lie in a bounded subset in $L^{2}\left((0, T) ;\left(H^{1}(\Omega)\right)^{*}\right)$. Hence, $w_{t}^{n} \rightarrow w_{t}^{*}$ and likewise for $s_{t}^{n}$. Using a Sobolev Embedding Theorem from Li and Yong [50], we know that $L^{2}\left((0, T) ;\left(H^{1}(\Omega)\right)\right.$ compactly embeds into $L^{2}(\Omega \times(0, T))$. Also, if $0<\epsilon<\frac{1}{2}$, then $L^{2}\left((0, T) ;\left(H^{1}(\Omega)\right)\right.$ also embeds into $L^{2}\left((0, T) ;\left(H^{\frac{1}{2}+\epsilon}(\Omega)\right)\right.$. With these two embeddings and using a comparison result in Simon [43], we have that $w^{n} \rightarrow w^{*}$ and $s^{n} \rightarrow s^{*}$ in $\mathrm{W}$ and in $L^{2}\left((0, T) ;\left(H^{\frac{1}{2}+\epsilon}(\Omega)\right)\right.$. By the continuous mapping of $H^{\frac{1}{2}+\epsilon}(\Omega) \rightarrow L^{2}(\partial \Omega)$, we have that $w^{n} \rightarrow w^{*}$ in $L^{2}\left((0, T) ; L^{2}(\partial \Omega)\right)$ and similarly for $s^{n}$. Moreover, since $\alpha^{n}$ and $\beta^{n}$ are $L^{\infty}$ bounded, there are subsequences $\alpha^{n}$ and $\beta^{n}$ such that $\alpha^{n} \rightarrow \alpha^{*}$ and $\beta^{n} \rightarrow \beta^{*}$ in $L^{2}\left((0, T) ; L^{2}(\partial \Omega)\right)$.

These convergences allow for us to pass to the limit in the weak formulation of the system (8-9). We find that $\left(w^{*}, s^{*}\right)$ is a weak solution associated with the control pair $\left(\alpha^{*}, \beta^{*}\right)$. Lastly, we use the lower semicontinuity of the functional $J(\alpha, \beta)$ with respect to weak convergences and recall, for example, that

$$
\varliminf_{n \rightarrow \infty} \int_{Q}\left(\alpha^{n}\right)^{2} d x d t \geq \int_{Q}\left(\alpha^{*}\right)^{2} d x d t
$$

to obtain that

$$
\begin{aligned}
J\left(\alpha^{*}, \beta^{*}\right) & =\int_{Q}\left\{K_{1} w^{*}+K_{2} s^{*}+\frac{1}{2} K_{3}\left(\alpha^{*}\right)^{2}+\frac{1}{2} K_{4}\left(\beta^{*}\right)^{2} d x d t\right. \\
& \leq \varliminf_{n \rightarrow \infty} \int_{Q}\left\{K_{1} w^{n}+K_{2} s^{n}+\frac{1}{2} K_{3}\left(\alpha^{n}\right)^{2}+\frac{1}{2} K_{4}\left(\beta^{n}\right)^{2} d x d t\right. \\
& \leq \lim _{n \rightarrow \infty} J\left(\alpha^{n}, \beta^{n}\right) \leq \min _{(\alpha, \beta) \in \mathcal{A}} J(\alpha, \beta) .
\end{aligned}
$$

Hence, we have that $\left(\alpha^{*}, \beta^{*}\right)$ is an optimal control pair that minimizes $J(\alpha, \beta)$.

\section{Optimality conditions}

To derive the representations of an optimal control pair, we must differentiate the functional with respect to the control pair. In order to accomplish this, we develop a linearized system referred to as a sensitivity system. This system is analyzed by considering the weak formulation of the system (4-5) with the controls augmented in a given direction. The mapping of the optimal control pair into the solution pair is differentiable as a directional derivative and converges to functions that satisfy a linearized system. Through the use of the sensitivity system we can determine the corresponding adjoint equations and then determine the optimality system that is composed of the state system coupled with the adjoint system along with the representations of the controls. 
Theorem 4.1. The mapping $(\alpha, \beta) \in \mathcal{A} \rightarrow(w(\alpha, \beta), s(\alpha, \beta)) \in W \times W$ is differentiable in the following sense:

$$
\begin{gathered}
\frac{w(\alpha, \beta+\epsilon h)-w(\alpha, \beta)}{h} \rightarrow \psi_{1} \text { in } W \\
\frac{s(\alpha, \beta+\epsilon h)-s(\alpha, \beta)}{h} \rightarrow \psi_{2} \text { in } W \\
\frac{w(\alpha+\epsilon l, \beta)-w(\alpha, \beta)}{l} \rightarrow \phi_{1} \text { in } W \\
\frac{s(\alpha+\epsilon l, \beta)-s(\alpha, \beta)}{l} \rightarrow \phi_{2} \text { in } W
\end{gathered}
$$

as $\epsilon \rightarrow 0$ for any $(\alpha, \beta) \in \mathcal{A}$ and $(h, l) \in\left(L^{\infty}\right)^{2}$ such that $(\alpha+\epsilon l, \beta+\epsilon h) \in \mathcal{A}$ for small $\epsilon$. Also, $\left(\psi_{1}, \psi_{2}\right)$ and $\left(\phi_{1}, \phi_{2}\right)$ satisfy

$$
\begin{aligned}
& \frac{\partial \psi_{1}}{\partial t}=\kappa \Delta \psi_{1}+\frac{\alpha w}{(1+w+s)^{2}}\left[2 \psi_{1}+w \psi_{1}-w \psi_{2}+2 s \psi_{1}\right]-\psi_{1} \mu(16) \\
& -\eta\left(2 w \psi_{1}+w \psi_{2}+s \psi_{1}\right) \\
& \frac{\partial \psi_{2}}{\partial t}=\kappa \Delta \psi_{2}-\psi_{2} \mu-\eta\left(s \psi_{1}+w \psi_{2}+2 s \psi_{2}\right)+h \\
& \frac{\partial \phi_{1}}{\partial t}=\kappa \Delta \phi_{1}+\frac{\alpha w}{(1+w+s)^{2}}\left[2 \phi_{1}+w \phi_{1}-w \phi_{2}+2 s \psi_{1}\right]-\phi_{1} \mu \\
& -\eta\left(2 w \phi_{1}+w \phi_{2}+s \phi_{1}\right)-\frac{l w^{2}}{1+w+s} \\
& \frac{\partial \phi_{2}}{\partial t}=\kappa \Delta \phi_{2}-\phi_{2} \mu-\eta\left(s \phi_{1}+w \phi_{2}+2 s \phi_{2}\right) \\
& \left(\begin{array}{l}
\psi_{1}(x, 0) \\
\psi_{2}(x, 0) \\
\phi_{1}(x, 0) \\
\phi_{2}(x, 0)
\end{array}\right)=\left(\begin{array}{l}
0 \\
0 \\
0 \\
0
\end{array}\right) \text { for } x \in \Omega \\
& \left(\begin{array}{c}
\nabla \psi_{1}(x, t) \cdot \nu+\psi_{1}(x, t) \\
\nabla \psi_{2}(x, t) \cdot \nu+\psi_{2}(x, t) \\
\nabla \phi_{1}(x, t) \cdot \nu+\phi_{1}(x, t) \\
\nabla \phi_{2}(x, t) \cdot \nu+\phi_{2}(x, t)
\end{array}\right)=\left(\begin{array}{l}
0 \\
0 \\
0 \\
0
\end{array}\right) \text { for } x \in \partial \Omega \text { and } t>0
\end{aligned}
$$

In order to obtain the necessary convergences, we utilize the weak form of the original system and obtain estimates similar to the procedure taken in the proof of the existence of an optimal control pair. For the details of a similar argument, we refer the reader to Fister and McCarthy [13] and Lions [28].

To derive the optimality system and to determine the characterizations of our controls, we must analyze the associated adjoint equations in addition to the associated adjoint operator for the system $\left(\psi_{1}, \psi_{2}\right)$ and $\left(\phi_{1}, \phi_{2}\right)$. First, we define

$$
\begin{aligned}
L_{1} w & =w_{t}-\kappa \Delta w \text { and } \\
L_{2} s & =w_{t}-\kappa \Delta s .
\end{aligned}
$$

For ease in notation, we define a matrix $M$ as

$$
M=\left(\begin{array}{cc}
\frac{-\alpha w}{(1+w+s)^{2}}[2+w+2 s]+\mu+2 \eta w & \frac{\alpha w}{(1+w+s)^{2}}+\eta(w+s) \\
\eta s & \mu+\eta(w+2 s)
\end{array}\right) .
$$


From our sensitivity Theorem 4.1, we can rewrite the equations in matrix notation. For example,

$$
\begin{aligned}
\mathcal{L}\left(\begin{array}{c}
\psi_{1} \\
\psi_{2}
\end{array}\right) & =\left(\begin{array}{l}
L_{1} \psi_{1}-\frac{\alpha w}{(1+w+s)^{2}}\left[2 \psi_{1}+w \psi_{1}-w \psi_{2}+2 s \psi_{1}\right]+\psi_{1} \mu+\eta\left(2 w \psi_{1}+w \psi_{2}+s \psi_{1}\right) \\
L_{2} \psi_{2}+\psi_{2} \mu+\eta\left(s \psi_{1}+w \psi_{2}+2 s \psi_{2}\right)
\end{array}\right) \\
& =\left(\begin{array}{l}
L_{1} \psi_{1} \\
L_{2} \psi_{2}
\end{array}\right)+M\left(\begin{array}{c}
\psi_{1} \\
\psi_{2}
\end{array}\right) \\
& =\left(\begin{array}{l}
0 \\
h
\end{array}\right) .
\end{aligned}
$$

With the same operator $L_{1}$ and the matrix, $M$, we see that

$$
\mathcal{L}\left(\begin{array}{l}
\phi_{1} \\
\phi_{2}
\end{array}\right)=\left(\begin{array}{l}
\frac{l w^{2}}{(1+w+s)} \\
0
\end{array}\right) .
$$

In order to devise the appropriate adjoint system, we let $(p, q)$ be the associated adjoint variable to our original system. To determine the optimality system, we recognize that

$$
\mathcal{L}^{*}\left(\begin{array}{c}
p \\
q
\end{array}\right)=\left(\begin{array}{l}
K_{1} \\
K_{3}
\end{array}\right),
$$

where the terms in the right hand matrix of the above equation are the partial derivatives of the integrand of the objective functional $J(\alpha, \beta)$ with respect to $u$ and then with respect to $w$. Additionally, the operator $L_{1}^{*}$ is the dual of operator $L_{1}$ as

$$
L_{1}^{*} p=-p_{t}-\kappa \Delta p
$$

Therefore, we have that

$$
\mathcal{L}^{*}\left(\begin{array}{c}
p \\
q
\end{array}\right)=\left(\begin{array}{c}
L_{1}^{*} p \\
L_{1}^{*} q
\end{array}\right)+M^{T}\left(\begin{array}{c}
p \\
q
\end{array}\right),
$$

in which $M^{T}$ is the transpose of the matrix $M$.

Using the notation just defined, we can now determine the characterizations of the optimal control pair, which will be denoted $\left(\alpha^{*}, \beta^{*}\right)$.

Theorem 4.2. Given $(\alpha, \beta) \in \mathcal{A}$ and the corresponding solutions $(w, s)$, there exists an adjoint pair $(p, q) \in$ $W \times W$ satisfying

$$
\begin{array}{r}
L_{1}^{*} p=K_{1}+\left(\frac{\alpha w(2+w+2 s)}{(1+w+s)^{2}}-\mu-2 \eta w\right) p-\eta s q \\
L_{1}^{*} q=K_{2}-\left(\frac{\alpha w(2+w+2 s)}{(1+w+s)^{2}}-\eta(w+s)\right) p-(\nu+\eta(w+2 s)) q \\
p(x, T)=q(x, T)=0 \text { for } x \in \Omega \text { and } \\
\nabla p(x, t) \cdot \nu+p(x, t)=\nabla q(x, t) \cdot \nu+q(x, t)=0 \text { on } \partial \Omega \times(0, T) .
\end{array}
$$

and

$$
\begin{aligned}
& \alpha^{*}=\min \left(\max \left(N_{1}, \frac{-p w^{2}}{K_{3}(1+w+s)}\right), N_{2}\right) \\
& \beta^{*}=\min \left(\max \left(M_{1}, \frac{q}{K_{4}}\right), M_{2}\right)
\end{aligned}
$$

Proof. For clearer understanding, we provide the proof associated with the characterization of the control $\alpha$. A similar argument produces the control representation for $\beta^{*}$. We suppose that $(\alpha, \beta)$ is an optimal control and $(w, s)$ is its corresponding solution pair. We assume that $(\alpha+\epsilon l, \beta) \in \mathcal{A}$ for $\epsilon>0$ with associated solution $\left(w_{\epsilon}, s_{\epsilon}\right)$ where $w_{\epsilon}=w(\alpha+\epsilon l, \beta)$ and $s_{\epsilon}=s(\alpha+\epsilon l, \beta)$. By a similar argument as in the existence proof for the solution, Theorem 2.2, we can obtain the existence of solutions to the system denoted in (27). 
Furthermore, work by Ladyzhenskaya et al. [22] and by Evans [10] provide existence proofs for similar state and adjoint systems.

We consider the directional derivative of $J(\alpha, \beta)$ with respect to $\alpha$ in the direction of $l$. Since the minimum value of $J(\alpha, \beta)$ is obtained, we assume it occurs at $(\alpha, \beta)$. Note that we are omitting the asterisk notation for simplicity. Through integration by parts, we use the relationship that

$$
\mathcal{L}^{*}\left(\begin{array}{c}
p \\
q
\end{array}\right)=\mathcal{L}\left(\begin{array}{c}
\phi_{1} \\
\phi_{2}
\end{array}\right)
$$

Therefore,

$$
\begin{aligned}
& \leq \lim _{\epsilon \rightarrow 0^{+}} \frac{J(\alpha+\epsilon l, \beta)-J(\alpha, \beta)}{\epsilon} \\
= & \lim _{\epsilon \rightarrow 0^{+}} \frac{1}{\epsilon} \int_{Q}\left(K_{1} w_{\epsilon}+K_{2} s_{\epsilon}+\frac{1}{2} K_{3}(\alpha+\epsilon l)^{2}+\frac{1}{2} K_{4} \beta^{2}-K_{1} w-K_{2} s-\frac{1}{2} K_{3} \alpha^{2}-\frac{1}{2} K_{4} \beta^{2}\right) d x d t \\
= & \int_{Q}\left(K_{1} \phi_{1}+K_{2} \phi_{2}+K_{3} \alpha l\right) d x d t \\
= & \int_{Q}\left(\left(\phi_{1}, \phi_{2}\right)\left(\begin{array}{c}
K_{1} \\
K_{2}
\end{array}\right)+K_{3} \alpha l\right) d x d t \\
= & \int_{Q}\left((p, q) \mathcal{L}^{*}\left(\begin{array}{c}
p \\
q
\end{array}\right)+K_{3} \alpha l\right) d x d t \\
= & \int_{Q}\left((p, q) \mathcal{L}\left(\begin{array}{c}
\phi_{1} \\
\phi_{2}
\end{array}\right)+K_{3} \alpha l\right) d x d t \\
= & \int_{Q}\left((p, q)\left(\begin{array}{c}
l w^{2} \\
0
\end{array}\right)+K_{3} \alpha l\right) d x d t \\
= & \int_{Q} l\left(\frac{p w^{2}}{(1+w+s)}+K_{3} \alpha\right) d x d t
\end{aligned}
$$

By standard optimality techniques $[23,28]$, we find that

$$
\alpha^{*}=\min \left(\max \left(M_{1}, \frac{-p w^{2}}{K_{3}(1+w+s)}\right), M_{2}\right) .
$$

Using a similar discussion with varying $\beta$ in the direction of $h$, we see that

$$
\beta^{*}=\min \left(\max \left(N_{1}, \frac{q}{K_{4}}\right), N_{2}\right) .
$$

The optimality system that is comprised of our state system (4-5) coupled with the adjoint system (27) through the characterization of an optimal control pair $\left(\alpha^{*}, \beta^{*}\right)$ and is denoted by the following system: 


$$
\begin{aligned}
\frac{\partial w}{\partial t} & =\kappa \Delta w+w\left[\frac{\alpha^{*} w}{1+w+s}-(\mu+\eta(w+s))\right] \\
\frac{\partial s}{\partial t} & =\kappa \Delta s-s(\mu+\eta(w+s))+\beta^{*} \\
-\frac{\partial p}{\partial t} & =\kappa \Delta p+K_{1}+\left(\frac{\alpha^{*} w(2+w+2 s)}{(1+w+s)^{2}}-\mu-2 \eta w\right) p-\eta s q \\
-\frac{\partial q}{\partial t} & =\kappa \Delta q+K_{2}-\left(\frac{\alpha^{*} w(2+w+2 s)}{(1+w+s)^{2}}-\eta(w+s)\right) p-(\mu+\eta(w+2 s)) q \\
w(x, 0) & =w_{0}(x) \text { and } s(x, 0)=s_{0}(x) \text { for } x \in \Omega \\
p(x, T) & =q(x, T)=0 \text { for } x \in \Omega \\
\nabla w(x, t) \cdot \nu & +w(x, t)=\nabla s(x, t) \cdot \nu+s(x, t)=0 \text { for } x \in \partial \Omega \text { and } t>0 \\
\nabla p(x, t) \cdot \nu & +p(x, t)=\nabla q(x, t) \cdot \nu+q(x, t)=0 \text { on } \partial \Omega \times(0, T) \\
\alpha^{*} & =\min \left(\max \left(M_{1}, \frac{-p w^{2}}{K_{3}(1+w+s)}\right), M_{2}\right) \\
\beta^{*} & =\min \left(\max \left(N_{1}, \frac{q}{K_{4}}\right), N_{2}\right) .
\end{aligned}
$$

We recognize that the uniqueness of the optimality solution, (30), will grant uniqueness of our optimal control pair since an optimal control pair and associated state and adjoint solutions satisfy the system (30). Moreover, the adjoint system (27) has corresponding final time conditions (transversality conditions) while the state system (4-5) has initial time conditions. Hence, the adjoint and state systems have opposite time orientations. Uniqueness of the optimal control pair can be determined but it is found for small final time, $\mathrm{T}$. We reference Fister [12] and a text by Lenhart and Workman [23] for details for the proof of the following stated theorem.

Theorem 4.3. For $T$ sufficiently small, the weak solution to the optimality system (30) is unique.

\section{Numerical Simulations}

For the numerical simulations discussed below, we set the parameter values based on a literature review for Anopheles gambiae, an African mosquito known to carry malaria. In our original model (1), $A$ represents the number of matings per day. Therefore $1 / A$ is the number of days to reproduce from egg to adult. $M$ represents the adult mosquito death rate and so $1 / M$ is the number of days as an adult. Lutambi et al., [29], Holsetein [18], and Service [41] provide an analysis of the life cycle that allows us to approximate $1 / A \approx 11$ days. Midega et al., [31], gives $M \approx 0.05$ days. According to Lutambi et al, [29] and [31], the diffusion rate can be estimated from daily flight ranges of $200-400 \mathrm{~m} /$ day which yields $D \approx 0.126-0.503 \mathrm{~km}^{2} /$ day. We set this to be $D=0.2 \mathrm{~km}^{2} /$ day. $K$ represents the density dependent death rate and is given by Lutambi et al. [29] as $K=0.05 / \mathrm{mosq} /$ day. The parameters are summarized in Table 5.

\begin{tabular}{crcr}
\hline Original Parameter & Value for Anopheles gambiae & Transformed Parameter & Transformed value \\
$D$ & 0.2 & $\kappa=D$ & 0.2 \\
$A$ & 0.09 & $\alpha=A$ & 0.09 \\
$M$ & 0.05 & $\mu=M \varepsilon$ & $0.05 \varepsilon$ \\
$K$ & 0.05 & $\eta=K \varepsilon$ & $0.05 \varepsilon$ \\
\hline
\end{tabular}

Table 1: Parameter values for Anopheles gambiae

By minimizing the objective functional

$$
J(\alpha, \beta)=\int_{Q}\left\{K_{1} w+K_{2} s+\frac{1}{2}\left(K_{3} \alpha^{2}+K_{4} \beta^{2}\right)\right\} d x d t
$$


we seek to simultaneously reduce the fecundity of the wild mosquitoes by minimizing the nonlinear cost $\alpha^{2}$ of the Holling II reproductive term $\alpha$ for the wild mosquitoes, the nonlinear cost of $\beta^{2}$ of the rate $\beta$ at which the sterile mosquitoes are being introduced, the total number of wild mosquitoes $w$ present over the time inverval $[0, T]$, and the total number of sterile mosquitoes $s$ present over the time interval $[0, T]$. We note that a value of $\alpha=0$ maximizes the impact of the larvicide by eliminating all growth of the wild mosquitoes $w$. Recall that here, and throughout the paper, we generally refer to habitat modification techniques as the use of larvicide. Recall that $\alpha$ and $\beta$ satisfy the bounds $M_{1} \leq \alpha(x, y, t) \leq M_{2}, N_{1} \leq \beta(x, y, t) \leq N_{2}$. We give equal priority to each of the terms in the objective functional and set $K_{i}=1$ for $i=1, \ldots, 4$.

We note that the parameter $\varepsilon$ is a measure of the density required for a constant mating rate. For small values of $\varepsilon$, the law of mass-action dominates the model and we are considering the case where $c(W, S) \approx$ $A(W+S)$. For larger values of $\varepsilon$, we are considering the constant mating case where $c(W, S) \approx A$.

In the optimality system (30), we note that the model equations move forward in time from an initial condition, while the adjoint equations move backward in time from a final condition. Typically, this type of problem is solved using an algorithm developed by Hackbusch [16] and recommended by Lenhart and Workman [23]. The algorithm generally converges only for small values of $T$. In order to allow the noncontrolled system to reach equilibrium, we needed to run the simulations for larger $T$ values. As a result we chose an alternative approach to the system that we found in literature related to the software COMSOL. Rather than treat the PDEs as separate equations that move forward and backward in time, we transform the coupled optimality system in the space-time cylinder to an elliptic system in three dimensional space, $[33,49]$.

Working in $x y$ space and letting $z=t$, our dimensionless optimality system becomes:

$$
\begin{aligned}
w_{z}-\kappa\left(w_{x x}+w_{y y}\right)-w\left[\frac{\alpha^{*} w}{1+w+s}-(\mu-\eta(w+s))\right] & =0 \\
s_{z}-\kappa\left(s_{x x}+s_{y y}\right)-s(\mu+\eta(w+s)) & =\beta^{*} \\
-p_{z}-\kappa\left(p_{x x}+p_{y y}\right)-\left(\frac{\alpha^{*} w(2+w+2 s)}{(1+w+s)^{2}}-\mu-2 \eta w\right) p+\eta s q & =K_{1} \\
-q_{z}-\kappa\left(q_{x x}+q_{y y}\right)+\left(\frac{\alpha^{*} w(2+w+2 s)}{(1+w+s)^{2}}+\eta(w+s)\right) p+(\mu+\eta(w+2 s)) q & =K_{2} \\
w(x, y, 0)=w_{0}(x, y) \text { and } s(x, y, 0)=s_{0}(x, y) \text { for }(x, y) \in \Omega & \\
p(x, y, T)=q(x, y, T)=0 \text { for }(x, y) \in \Omega & \\
\nabla_{x y} w(x, y, z) \cdot \nu+w(x, y, z)=\nabla_{x y} s(x, y, z) \cdot \nu+s(x, y, z)=0 \text { on } \partial \Omega \times(0, T) & \\
\nabla_{x y} p(x, y, z) \cdot \nu+p(x, y, z)=\nabla_{x y} q(x, y, z) \cdot \nu+q(x, y, z)=0 \text { on } \partial \Omega \times(0, T) & \\
\alpha^{*}=\min \left(\max \left(M_{1}, \frac{-p w^{2}}{K_{3}(1+w+s)}\right), M_{2}\right) & \\
\beta^{*}=\min \left(\max \left(N_{1}, \frac{q}{K_{4}}\right), N_{2}\right) . &
\end{aligned}
$$

This is an elliptic system on a cylinder $\Omega \times(0, T)$. We solve this numerically using COMSOL, a finiteelement software.

\section{1. $C A S E$ 1: $\varepsilon=0.01$}

The parameter $\varepsilon$ is a measure of the density required for a constant mating rate. For this small value of $\varepsilon$, the law of mass-action dominates the model and we are considering the case where $c(W, S) \approx A(W+S)$.

\subsubsection{No larvicide with no sterile mosquito release}

We begin by considering the interaction of wild and sterile mosquitoes in the absence of larvicide, $\alpha=$ $A=0.09$ and without additional release of sterile insects, $\beta=0$. In this case $J(\alpha, \beta)=\int_{Q}\left\{w+\frac{1}{2} \alpha^{2}\right\} d x d t$, which amounts to minimizing the total number of mosquitoes in the absence of control variables. Horizontal and vertical slices are shown of the $x y t$ cylinder for $0 \leq x^{2}+y^{2} \leq 1,0 \leq t \leq 5$. For both the wild and sterile mosquito populations, the population can be seen to decay over time. We note that the sterile population decays more rapidly. 


\subsubsection{No larvicide with sterile mosquito release}

We now allow sterile mosquito release in the absence of larvicide, $\alpha=0.09$ and $N_{1} \leq \beta \leq N_{2}$. We note that in Figure 3, we can see that the wild mosquito population decreases quite rapidly. In Figure 4, we see that the sterile mosquito population decreases more slowly, because the sterile release continues for some time as shown in Figure 5.

\subsubsection{Larvicide with no sterile mosquito release}

We now allow the use of larvicide in the absence sterile mosquito release, $M_{1} \leq \alpha \leq M_{2}$ and $\beta=0$. We set $M_{2}=A=0.09$ to reflect the maximum number of matings per day before reducing it via optimal control. The control $\alpha^{*}$ attains the lower bound $M_{1}=0.01$ and in Figures 6 and 7, we see that both the wild and the sterile mosquito populations are eliminated through maximal use of larvicide slightly more rapidly thatn in the case where $\alpha=0.09$.

\subsubsection{Mixture of strategies}

We now allow sterile mosquito release with the use of larvicide, $M_{1} \leq \alpha \leq M_{2}$ and $N_{1} \leq \beta \leq N_{2}$. In Figures 8 and 9, we see that the wild mosquito populations are gradually eliminated. With continual introduction of the sterile mosquitoes, their population will stabilize. The larvicide control $\alpha^{*}$ attains the lower bound 0.01 and the sterile release control $\beta^{*}$ gradually decays to zero as shown in Figure 10. Note that there is not much difference between this case and the case with no larvicide and with sterile mosquito release.

\section{2. $C A S E$ 2: $\varepsilon=10$}

For this larger value of $\varepsilon$, we are considering the constant mating case where $c(W, S) \approx A$. The wild and sterile mosquito populations decay much more repidly for this model than for the small $\varepsilon$ case. This is because the contribution of the mating term is smaller. All plots in this section are over the interval $0 \leq t \leq 2$.

\subsubsection{No larvicide with no sterile mosquito release}

Once again, we consider the interaction of wild and sterile mosquitoes in the absence of larvicide, $\alpha=$ $A=0.09$ and without additional release of sterile insects, $\beta=0$. In practice, we minimize the total number of mosquitoes in the absence of control variables. Horizontal and vertical slices are shown of the $x y t$ cylinder for $0 \leq x^{2}+y^{2} \leq 1,0 \leq t \leq 2$. For both the wild and sterile mosquito populations, the population can be seen to decay over time much more rapidly than for $\varepsilon=0.01$, see Figures 11 and 12 . We note that the sterile and wild populations decay at comparable rates in this case.

\subsubsection{No larvicide with sterile mosquito release}

We now allow sterile mosquito release in the absence of larvicide, $\alpha=0.09$ and $N_{1} \leq \beta \leq N_{2}$. We note that in Figures 13 and 14, we can see that the wild and sterile mosquito populations decrease quite rapidly. In Figure 15, the optimal sterile release peaks midway through the time interval before returning to zero once the wild an sterile populations have been eliminated.

\subsubsection{Larvicide with no sterile mosquito release}

\subsubsection{Mixture of strategies}

We now allow sterile mosquito release with the use of larvicide, $M_{1} \leq \alpha \leq M_{2}$ and $N_{1} \leq \beta \leq N_{2}$. In Figures 18 and 19, we see that both the wild and sterile mosquito populations are eliminated more quickly than in the small $\varepsilon$ case. The larvicide control $\alpha^{*}$ attains the lower bound 0.01 and the sterile release control $\beta^{*}$ starts close to zero, increases to approximately 0.4 and the decreases to zero again as shown in Figure 20. The fundamental difference between this case and the small $\varepsilon$ case is that the sterile release $\beta$ does not need to persist because the wild and sterile mosquito populations have already been eliminated. 


\section{Discussion}

This paper describes an optimal control approach to mosquito population reduction in the presence of diffusion. It is an extension of our previous work [14]. We model the reproductive term using a HollingII functional response, [17, 26], and we note this allows us to simultaneously model the mating dynamics of both small and large populations. We have demonstrated that it is possible, through optimal choices of larvicide use (or habitat modification) and/or sterile insect release, to eliminate the wild mosquito population. The effect of the parameter $\varepsilon$ is subtantial. For larger value of $\varepsilon$, we are considering the constant mating case where $c(W, S) \approx A$, while for small $\varepsilon$ values, we consider the case where $c(W, S) \approx A(W+S)$. The wild and sterile mosquito populations decay much more rapidly for this model for large values of $\varepsilon$, i.e. the constant mating case.

Overall, our findings are that if larvicide is allowed, it should be used at a maximal level $\alpha^{*}=M_{1}$, and that a combination of the techniques leads to a more rapid elimination of the wild mosquito population. We note that other authors have found similar results using other models $[2,7,8,9,46]$. Dumont and Tcheuenche [8] found that the $R_{0}$ of a disease could be reduced through a combination of periodic sterile insect release and habitat modification. By capturing the dynamics of both large and small mosquito populations, we can get a sense of whether larvicide or sterile insect release is best for each situation. Our numerical results suggest that a combination of both strategies is optimal when they are comparably weighted in the objective functional.

\section{Acknowledgements}

We acknowledge that Fister and McCarthy's work on this project has been made possible through a grant from the National Science Foundation, DUE-1028125.we thank Jerome Goddard of the Department of Biochemistry, Molecular Biology, Entomology, and Plant Pathology of Mississippi State University for useful conversations.
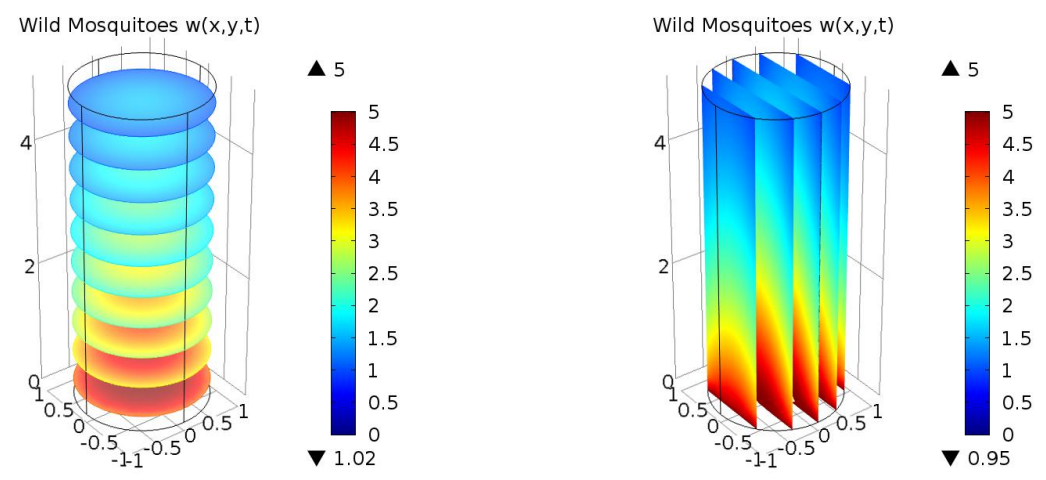

Figure 1: Wild Mosquitoes, $\varepsilon=0.01$ with bounds $\alpha(t)=0.09, \beta(t)=0$, initial conditions $w_{0}=5, s_{0}=5$. The horizontal plane is the $x y$-plane on the unit circle and the vertical is the $t$-direction for times $t=[0,5]$. 

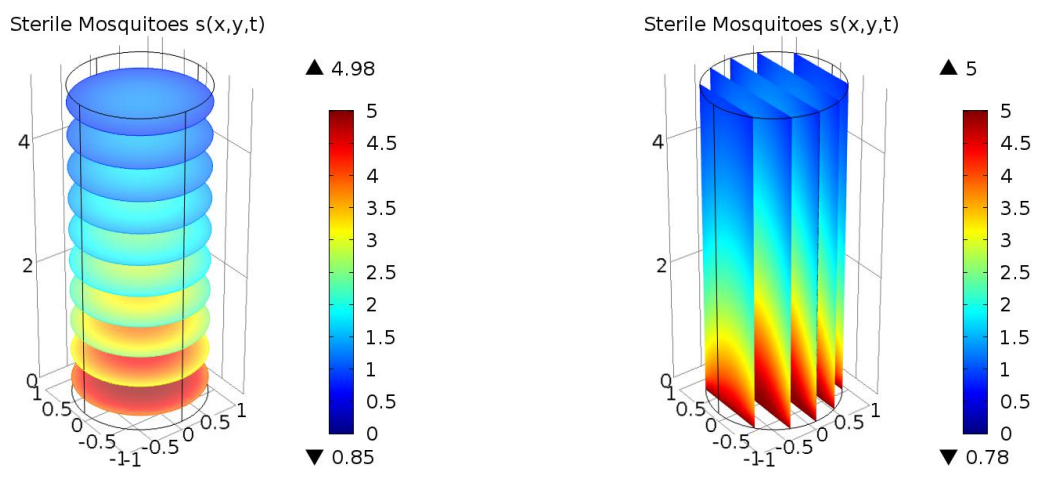

Figure 2: Sterile Mosquitoes, $\varepsilon=0.01$ with bounds $\alpha(t)=0.09, \beta(t)=0$, initial conditions $w_{0}=5, s_{0}=5$. The horizontal plane is the $x y$-plane on the unit circle and the vertical is the $t$-direction for times $t=[0,5]$.
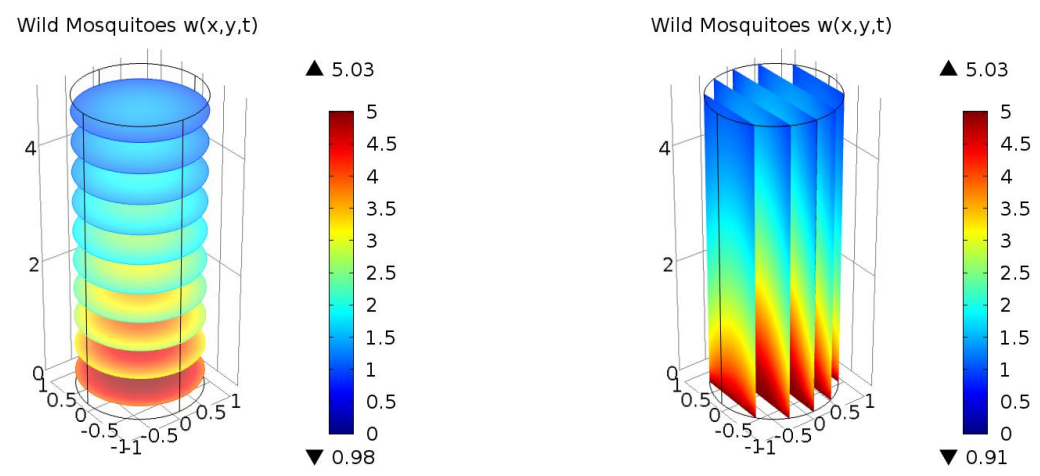

Figure 3: Wild Mosquito population $\varepsilon=0.01$ with $\alpha(t)=0.09,0 \leq \beta(t) \leq 1$, initial conditions $w_{0}=5, s_{0}=5$. The horizontal plane is the $x y$-plane on the unit circle and the vertical is the $t$-direction for times $t=[0,5]$.

\section{References}

[1] Alford, J.G. A reaction-diffusion model of the Darien Gap Sterile Insect Release Method. Communications in Nonlinear Science and Numerical Simulation, 22 (1-3), 175-185, 2015.

[2] L. Alphey, L. Alphey and M.B. Bonsall A Model Framework to Estimate Impact and Cost of GeneticsBased Sterile Insect Methods for Dengue Vector Control. PLOS One, 6 (10), 2011.

[3] L. Alphey, M. Benedict, R. Bellini, G.G. Clark, D.A. Dame, M.W. Service and S.L. Dobson. SterileInsect Methods for Control of Mosquito-Borne Diseases: An Analysis. Vector-Borne and Zoonotic Diseases, 10: 295-311, 2010.

[4] H. Barclay. Mathematical Models for the Use of Sterile Insects in: V. Dyck, J. Hendrichs, and A.S. Robinson (Eds.), Sterile Instect Technique, Springer, 147-174, 2005.

[5] A.C. Bartlett Insect sterility, insect genetics, and insect control in: D. Pimentel (Ed.), Handbook of Pest Management in Agriculture, Vol. II., CRC Press, Boca Raton, FL, 2:279-287, 1990.

[6] L. Cai, S. Ai, and J. Li Dynamics of Mosquitoes Populations with Different Strategies for Releasing Sterile Mosquitoes. SIAM Journal on Applied Mathematics , 74(6), 1786-1809, 2014.

[7] H. Diaz, A.A. Ramirez, A. Olarte, C. Clavijo. A model for the control of malaria using genetically modified vectors Journal of Theoretical Biology, 276: 57-66, 2011. 

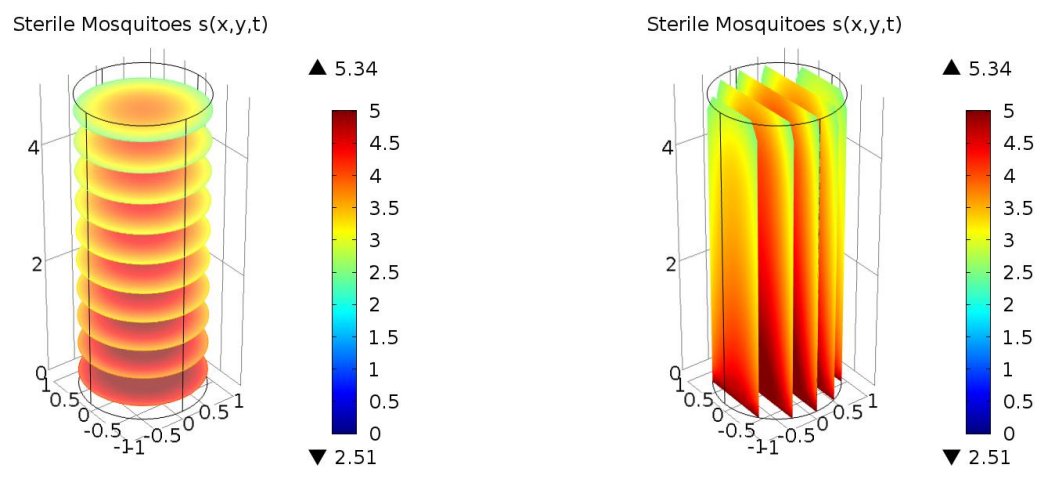

Figure 4: Sterile Mosquito population $\varepsilon=0.01$ with $\alpha(t)=0.09,0 \leq \beta(t) \leq 1$, initial conditions $w_{0}=5, s_{0}=5$. The horizontal plane is the $x y$-plane on the unit circle and the vertical is the $t$-direction for times $t=[0,5]$.
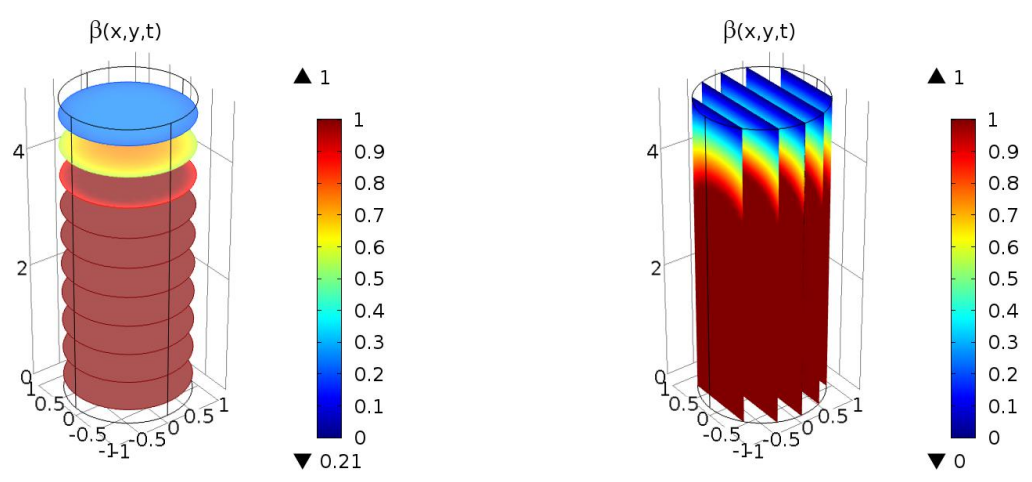

Figure 5: Optimal sterile mosquito release $\beta^{*}$ for $\varepsilon=0.01$ with $\alpha(t)=0.09,0 \leq \beta(t) \leq 1$, initial conditions $w_{0}=5, s_{0}=5$. The horizontal plane is the $x y$-plane on the unit circle and the vertical is the $t$-direction for times $t=[0,5]$.

[8] Y. Dumont and J.M. Tchuenche. Mathematical studies on the sterile insect technique for the Chikungunya disease and Aedes albopictus. J. Mathematical Biology, 65: 809-854, 2012.

[9] L. Esteva and H.M. Yang. Mathematical model to assess the control of Aedes aegypti mosquitoes by the sterile insect technique. newblock Mathematical Biosciences, 198: 132-147, 2005.

[10] L.C. Evans. Partial differential equations. American Mathematical Society, Providence, RI 1998.

[11] R.W. Fay, E.M. McCray, Jr., and J.W. Kirkpatrick. Mass production of sterilized male Aedes aegypti. Mosquito News, 23, 210-214, 1963.

[12] K.R. Fister Optimal control of harvesting in a predator-prey parabolic system. Houston Journal of Mathematics , 23:2, 341-355, 1997.

[13] K.R. Fister and M.L. McCarthy. Optimal control of a chemotaxis system. Quarterly of Applied Mathematics, LXI, 193-211, 2003.

[14] K.R. Fister, M.L. McCarthy, S.F. Oppenheimer and C. Collins Optimal control of insects through sterile release and habitat modification. Mathematical Biosciences, 244, 201-12, 2013.

[15] H. Gaff, D. Hartley, N. Leahy. An Epidemiological Model of Rift Valley Fever Electronic Journal of Differential Equations,(115): 1-12, 2007.

[16] W.K. Hackbusch. A numerical method for solving parabolic equations with opposite orientations. Computing, 20:229-240, 1978. 

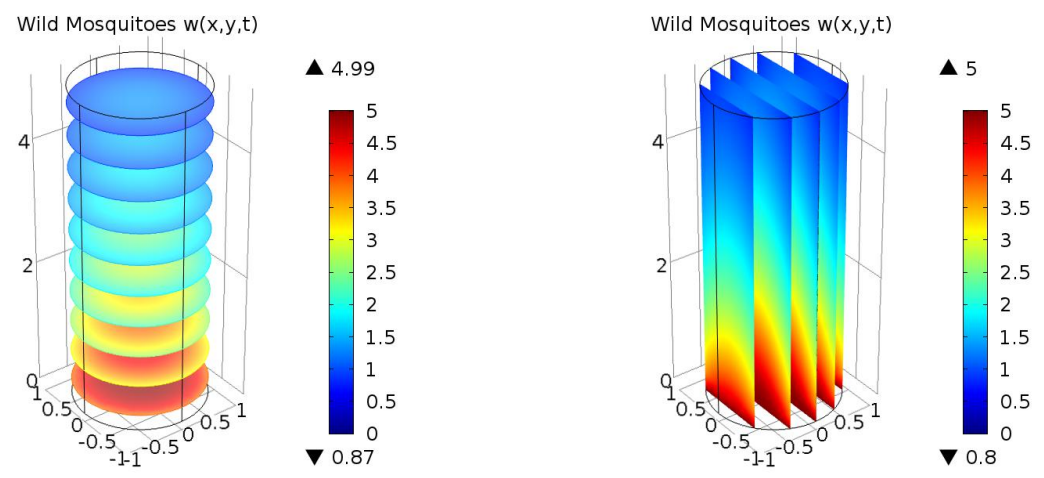

Figure 6: Wild Mosquito population $\varepsilon=0.01$ with bounds $0.01 \leq \alpha(t) \leq 0.09, \beta(t)=0$, initial conditions $w_{0}=5, s_{0}=5$. The horizontal plane is the $x y$-plane on the unit circle and the vertical is the $t$-direction for times $t=[0,5]$.
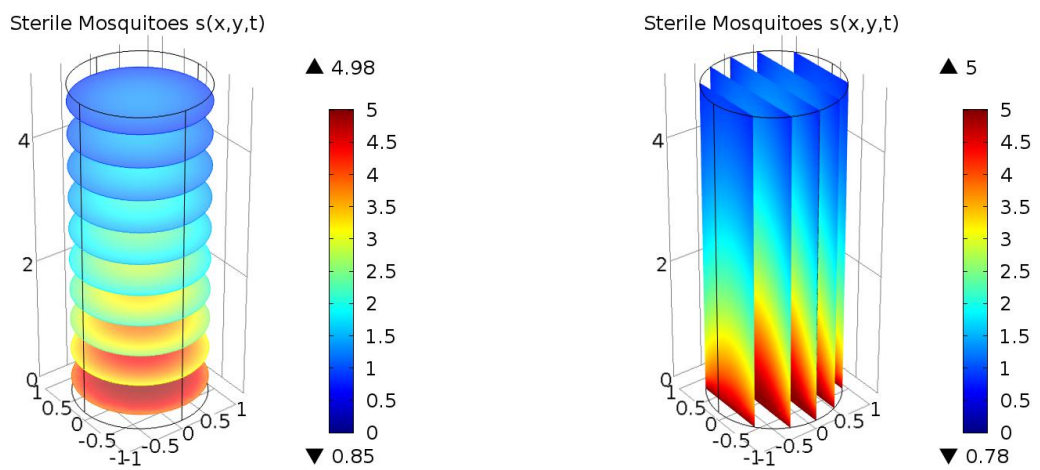

Figure 7: Sterile Mosquito population $\varepsilon 0.01$ with bounds $0.01 \leq \alpha(t) \leq 0.09, \beta(t)=0$, initial conditions $w_{0}=5, s_{0}=5$. The horizontal plane is the $x y$-plane on the unit circle and the vertical is the $t$-direction for times $t=[0,5]$.

[17] C.S. Holling. The components of predation as revealed by a study of small mammal predation of the European pine sawfly. Canad. Entomol., 91:293-320, 1959.

[18] M. H. Holsetein. Biology of Anopheles Gambiae: research in French West Africa. Monograph series number 9, World Health Organization, Palais des Nations, Geneva. 1954.

[19] E.F. Knipling. Possibilities of insect control or erradication through the use of sexually sterile males $J$. Econ. Entomol., 48: 459-462, 1955.

[20] E.F. Knipling. The basic principles of insect population suppression and management Agriculture Handbook 512. US Dept. of Agriculture, Washington DC, 1979.

[21] E.F. Knipling. Sterile insect technique as a screwworm control measure: the concept and its development, in: O.H. Graham (Ed.), Symposium on Eradication of the Screwworm from the United States and Mexico, Misc. Publ. Entomol. Soc. America, 62:1-4, 1985.

[22] O.A. Ladyzhenskaya, V.A. Solonnikov, and N.N. Ural'Tseva. Linear and quasi-linear equations of parabolic type. Nauka, Moscow, 1967; English transl., American Mathematical Society, Providence, RI, 1968.

[23] S. Lenhart and J.T. Workman Optimal Control Applied to Biological Models. Chapman \& Hall/CRC, 2007.

[24] M.A. Lewis and P. van den Driessche. Waves of Extinction from Sterile Insect Release. Mathematical Biosciences, 116:221-247, 1993. 

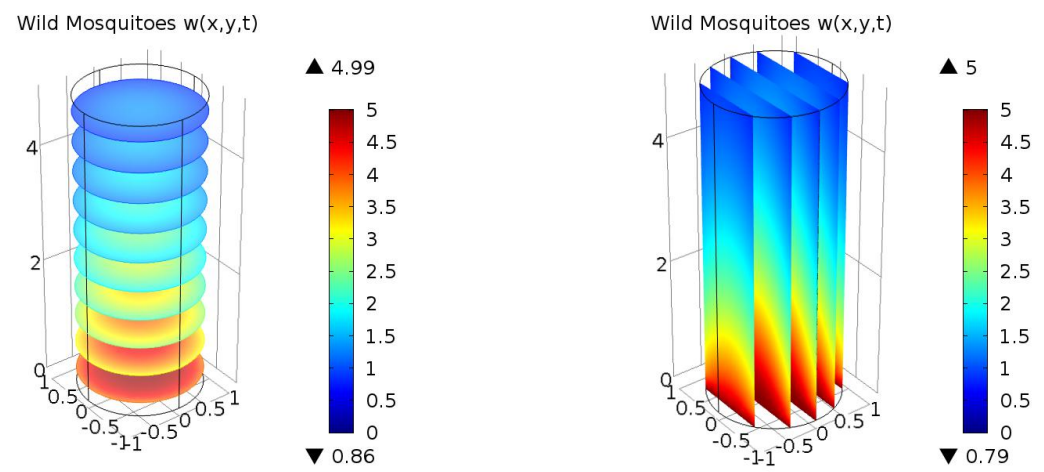

Figure 8: Wild Mosquito population $\varepsilon=0.01$ with bounds $0.01 \leq \alpha(t) \leq 0.09,0 \leq \beta(t) \leq 1$, initial conditions $w_{0}=5, s_{0}=5$. The horizontal plane is the $x y$-plane on the unit circle and the vertical is the $t$-direction for times $t=[0,5]$.
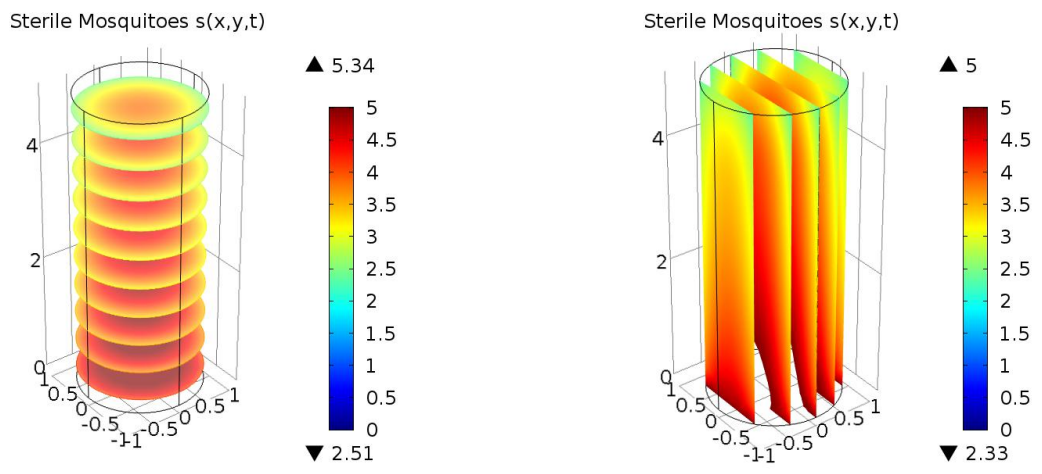

Figure 9: Sterile Mosquito population $\varepsilon=0.01$ with bounds $0.01 \leq \alpha(t) \leq 0.09,0 \leq \beta(t) \leq 1$, initial conditions $w_{0}=5, s_{0}=5$. The horizontal plane is the $x y$-plane on the unit circle and the vertical is the $t$-direction for times $t=[0,5]$.

[25] J. Li. Simple mathematical models for interacting wild and transgenic mosquito populations. Mathematical Biosciences, 189: 39-59, 2004.

[26] Jia Li. Differential equations models for interacting wild and transgenic mosquito populations. Journal of Biological Dynamics, 2(3):241-258, 2008.

[27] Jia Li and Zhiling Yuan. Modeling releases of sterile mosquitoes with different strategies. Journal of Biological Dynamics, 9(1):1-14, 2015.

[28] J.L. Lions. Optimal control of systems governed by partial differential equations. Springer-Verlag, New York, 1971.

[29] A. M. Lutambi, M. A. Penny, T. Smith and N. Chitnis. Mathematical modelling of mosquito dispersal in a heterogeneous environment. Mathematical Biosciences, 241, 198-216, 2013.

[30] E. Michael, M. Malecela-Lazaro, B. Maegga, P. Fischer, J. Kazura. Mathematical Models and Lymphatic Filariasis Control: Monitoring and Evaluating Interventions. TRENDS in Parasitology, 22(11): $529-535,2006$.

[31] J.T. Midega, C.M. Mbogo, H. Mwambi, M.D. Wilson, G. Ojwang, J.M. Mwangangi, J.G. Nzovu, J.I. Githure, G. Yan and C. Beier. Estimating dispersal and survival of Anopheles gambiae and Anopheles funestus along the Kenyan coast by using mark-release-recapture methods. Journal of Medical Entomology, 6 (44), 2007. 

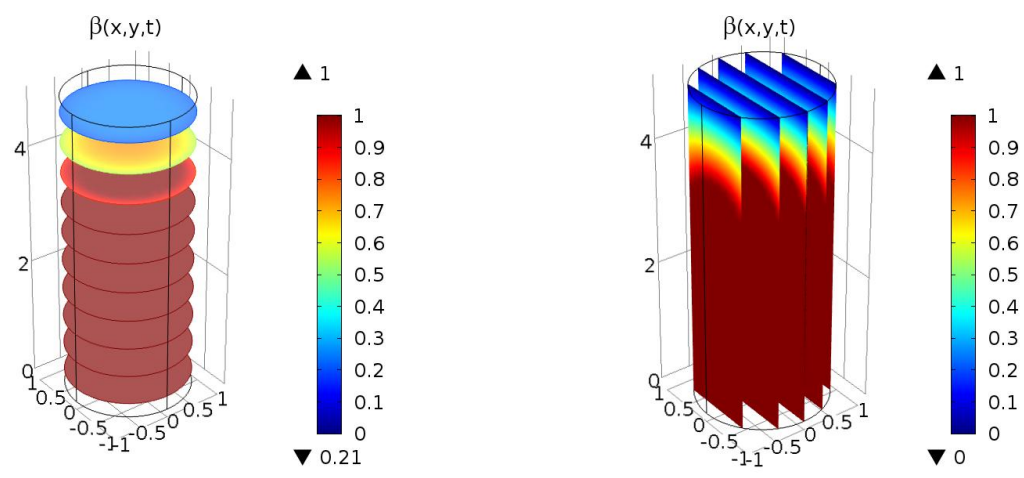

Figure 10: Optimal sterile mosquito release $\beta^{*}$ for $\varepsilon=0.01$ with bounds $0.01 \leq \alpha(t) \leq 0.09,0 \leq \beta(t) \leq 1$, initial conditions $w_{0}=5, s_{0}=5$. The horizontal plane is the $x y$-plane on the unit circle and the vertical is the $t$-direction for times $t=[0,5]$.
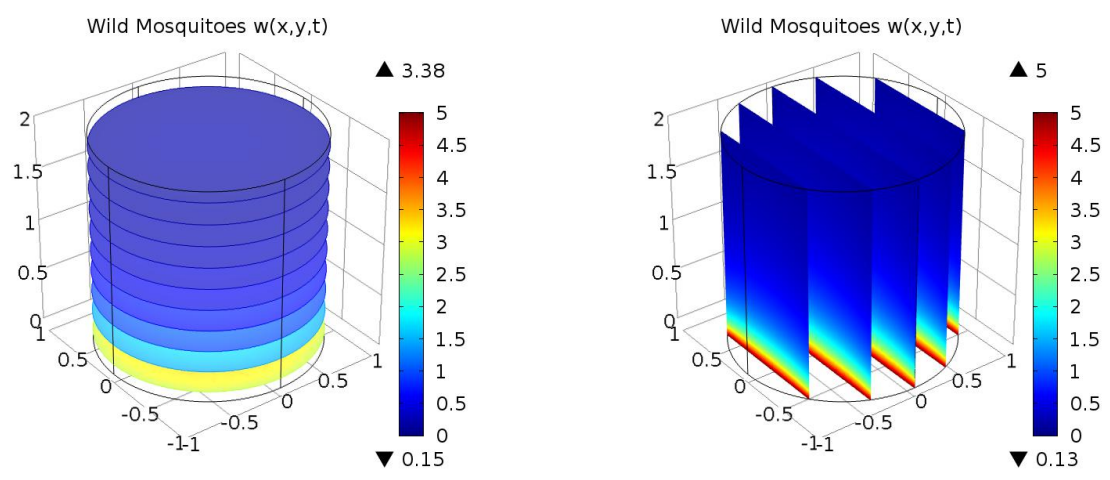

Figure 11: Wild Mosquitoes, $\varepsilon=10$ with bounds $\alpha(t)=0.09, \beta(t)=0$, initial conditions $w_{0}=5, s_{0}=5$. The horizontal plane is the $x y$-plane on the unit circle and the vertical is the $t$-direction for times $t=[0,2]$.

[32] H.B. Morlan , E. M. McCray, Jr., and J. W. Kilpatrick. Field tests for with sexually sterile males for control of Aedes aegypti. Mosq. News, 22(3):295-300, 1962.

[33] I. Neitzel, U. Prúfert and T. Slawig. Solving time-dependent optimal control problems in COMSOL multiphysics. Proceedings of the COMSOL Conference, Hanover, 2008.

[34] E. Newton and P. Reiter. A Model of the Transmission of Dengue Fever with an Evaluation of the Impact of Ultra-Low Volume (ULV) Insecticide Applications on Dengue Epidemics. American Journal of Tropical Hygiene and Medicine, 47(6): 709 -720, 1992.

[35] M. Oteroa, H. Solaria, N. Schweigmann. A Stochastic Population Dynamics Model for Aedes Aegypti: Formulation and Application to a City with Temperate Climate. Bulletin of Mathematical Biology, 2006(68): 1945-1974, 2006.

[36] A. Pazy. Semigroups of Linear Operators and Applications to Partial Differential Equations. SpringerVerlag, New York, 1983.

[37] R.E. Plant and M. Mangel. Modeling and Simulation in Agricultural Pest Management. SIAM Review, 29 (2):235-261, 1987.

[38] M. Predescu, G. Sirbu, R. Levins, T. Awerbuch-Friedlander. On the Dynamics of a Deterministic and Stochastic Model for Mosquito Control. Applied Mathematics Letters, 20: 919-925, 2007.

[39] M. Rafikov, L. Bevilacqua, A.P.P. Wyse. Optimal control strategy of malaria vector using genetically modified mosquitoes Journal of Theoretical Biology, 258: 418-425, 2009. 

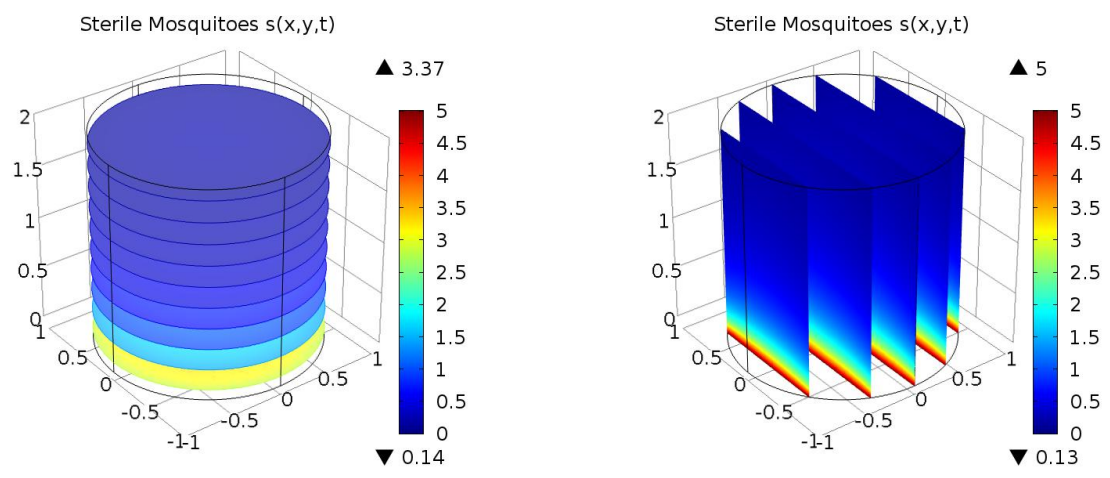

Figure 12: Sterile Mosquitoes, $\varepsilon=10$ with bounds $\alpha(t)=0.09, \beta(t)=0$, initial conditions $w_{0}=5, s_{0}=5$. The horizontal plane is the $x y$-plane on the unit circle and the vertical is the $t$-direction for times $t=[0,2]$.
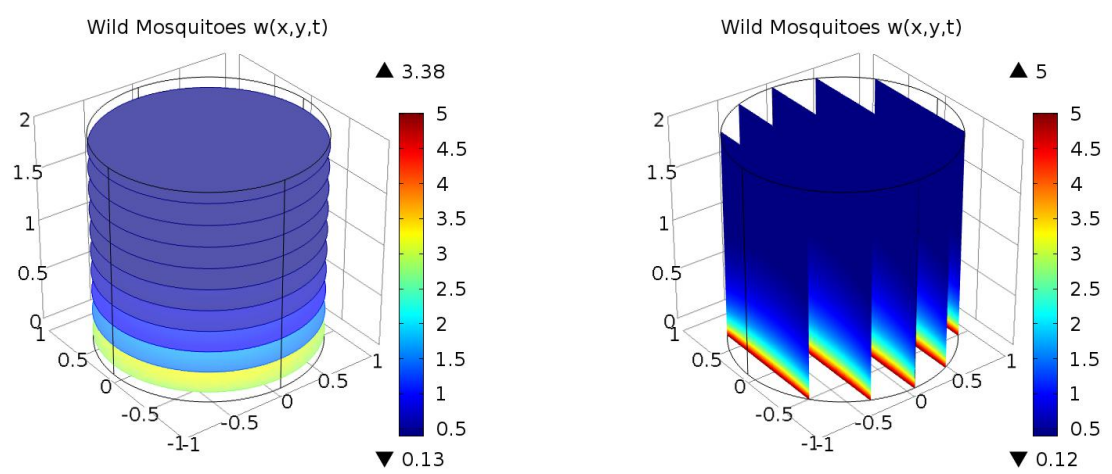

Figure 13: Wild Mosquito population $\varepsilon=10$ with $\alpha(t)=0.09,0 \leq \beta(t) \leq 1$, initial conditions $w_{0}=5, s_{0}=5$. The horizontal plane is the $x y$-plane on the unit circle and the vertical is the $t$-direction for times $t=[0,2]$.

[40] M. Rafikov, A.P.P. Wyse and L. Bevilacqua. Controlling the interaction between wild and transgenic mopsquitoes. Journal of Nonlinear Systems and Applications, 27-31, 2010.

[41] M.W. Service. Medical Entomology for Students. Cambridge University Press, Fifth Edition, 2012.

[42] R.E. Showalter. Hilbert Space Methods in Partial Differential Equations. Dover Publications, 2010.

[43] J. Simon, J. Compact sets in the space $L^{p}(0, T ; B)$. Annali di Matematica pura ed pplicata, CXLVI, No. IV, 1987, 65-96.

[44] C.N. Smith. Possible use of the sterile-male technique for control of Aedes Aegypti. Bull. Wld. Hlth. Org., 36, 633-635, 1967.

[45] D. Smith, J. Dushoff, F.E. McKenzie. The Risk of a Mosquito-Borne Infection in a Heterogeneous Environment. PLoS Biology, 2(11): 1957-1964, 2007.

[46] R.C.A. Thome, H.M. Yang and L. Esteva. Optimal control of Aedes aegypti mosquitoes by the sterile insect technique and insecticide. Mathematical Biosciences, 223: 12-23, 2010.

[47] A. Wyse, A., L. Bevilacqua, M. Rafikov. Simulating Malaria Model for Different Treatment Intensities in a Variable Environment. Ecological Modelling, 206: 322-330, 2007.

[48] L. Yakob, L. Alphey, M. Bonsall. Aedes aegypti Control: The Concomitant Role of Competition, Space and Transgenic Technologies. Journal of Applied Ecology, 45: 1258-1265, 2008. 

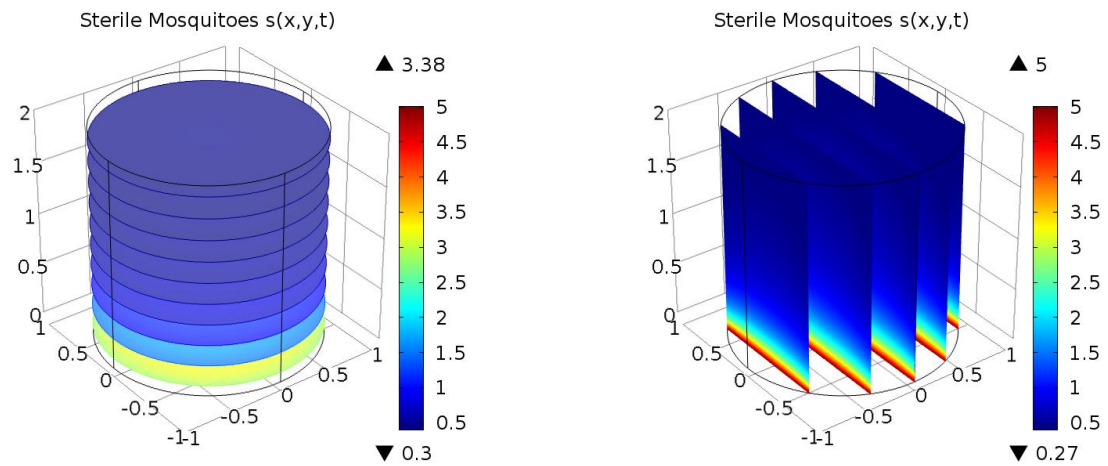

Figure 14: Sterile Mosquito population $\varepsilon=10$ with $\alpha(t)=0.09,0 \leq \beta(t) \leq 1$, initial conditions $w_{0}=5, s_{0}=5$. The horizontal plane is the $x y$-plane on the unit circle and the vertical is the $t$-direction for times $t=[0,2]$.
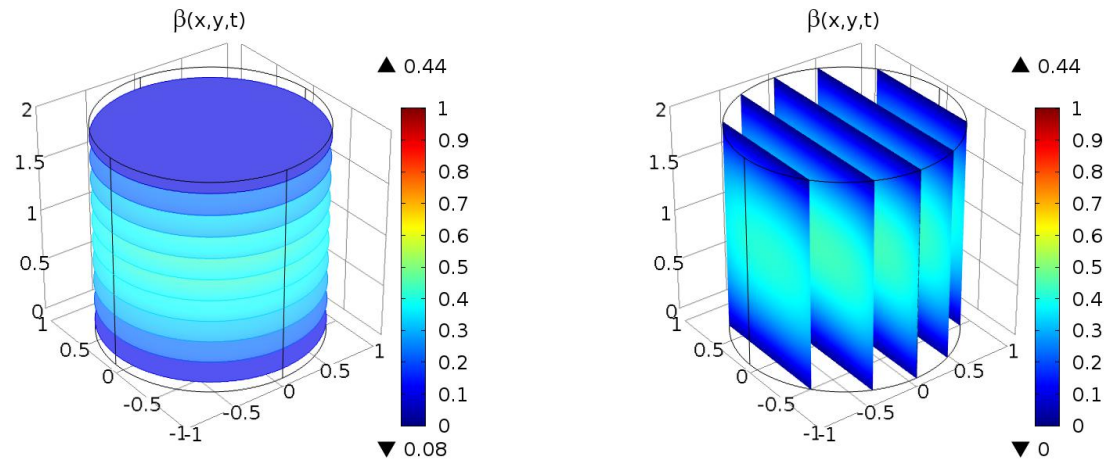

Figure 15: Optimal sterile mosquito release $\beta^{*}$ for $\varepsilon=10$ with $\alpha(t)=0.09,0 \leq \beta(t) \leq 1$, initial conditions $w_{0}=5, s_{0}=5$. The horizontal plane is the $x y$-plane on the unit circle and the vertical is the $t$-direction for times $t=[0,2]$.

[49] F. Yilmaz and B. Karas'ozen. Solving Distributed Optimal Control Problems for the Unsteady Burgers Equation in COMSOL Multiphysics. Proceedings of the COMSOL Conference, Milan, 2009.

[50] X. Li, J. Yong. Optimal control theory for infinite dimensional systems. Birkhauser, Boston, MA, 1995. 

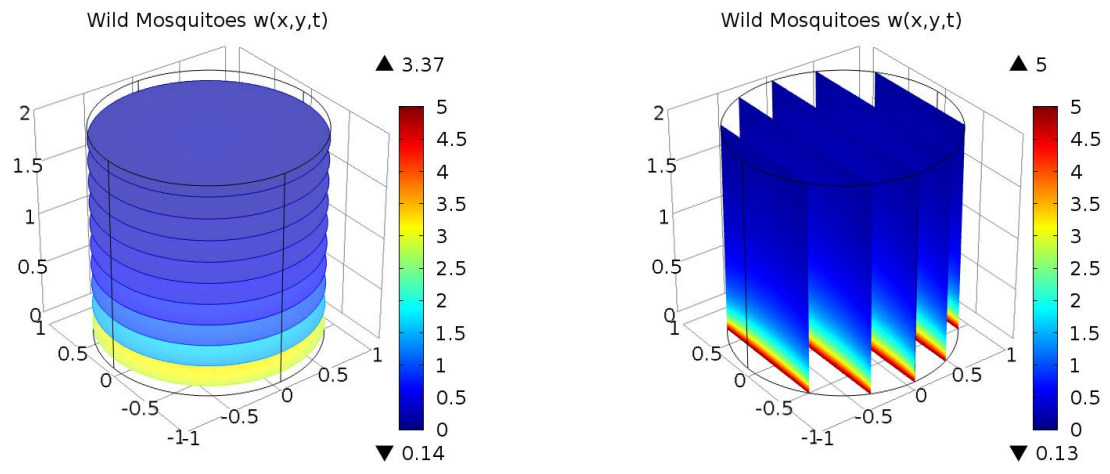

Figure 16: Wild Mosquito population $\varepsilon=10$ with bounds $0.01 \leq \alpha(t) \leq 0.09, \beta(t)=0$, initial conditions $w_{0}=5, s_{0}=5$. The horizontal plane is the $x y$-plane on the unit circle and the vertical is the $t$-direction for times $t=[0,2]$.
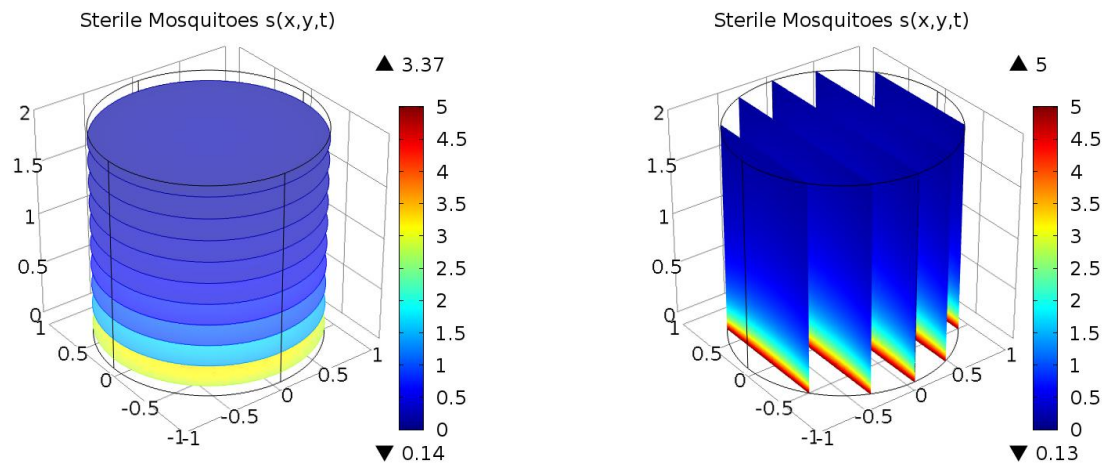

Figure 17: Sterile Mosquito population $\varepsilon=10$ with bounds $0.01 \leq \alpha(t) \leq 0.09, \beta(t)=0$, initial conditions $w_{0}=5, s_{0}=5$. The horizontal plane is the $x y$-plane on the unit circle and the vertical is the $t$-direction for times $t=[0,2]$.
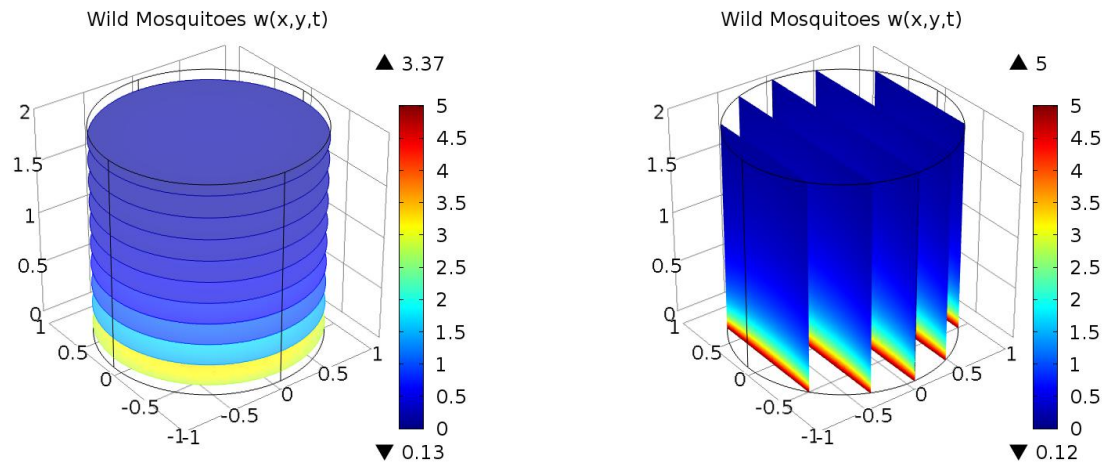

Figure 18: Wild Mosquito population $\varepsilon=10$ with bounds $0.01 \leq \alpha(t) \leq 0.09,0 \leq \beta(t) \leq 1$, initial conditions $w_{0}=5, s_{0}=5$. The horizontal plane is the $x y$-plane on the unit circle and the vertical is the $t$-direction for times $t=[0,5]$. 

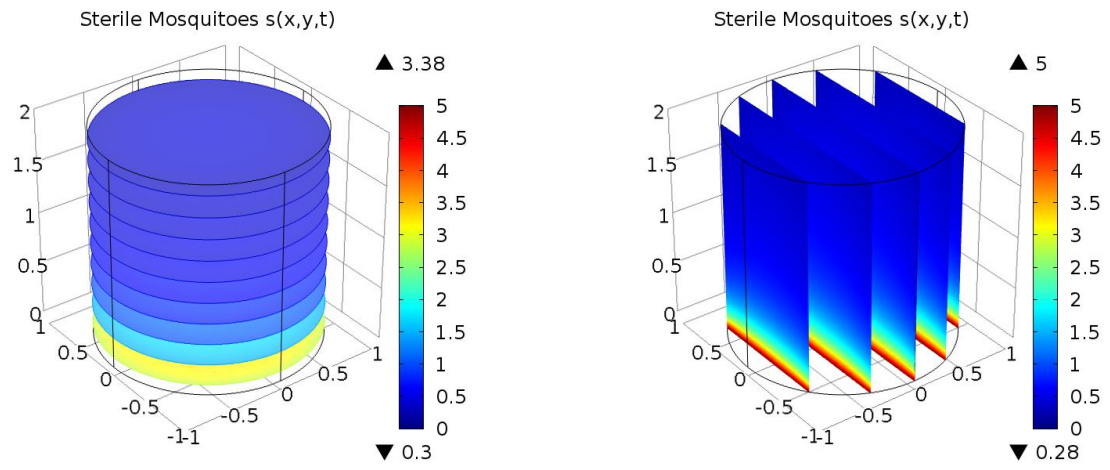

Figure 19: Sterile Mosquito population $\varepsilon=10$ with bounds $0.01 \leq \alpha(t) \leq 0.09,0 \leq \beta(t) \leq 1$, initial conditions $w_{0}=5, s_{0}=5$. The horizontal plane is the $x y$-plane on the unit circle and the vertical is the $t$-direction for times $t=[0,5]$.
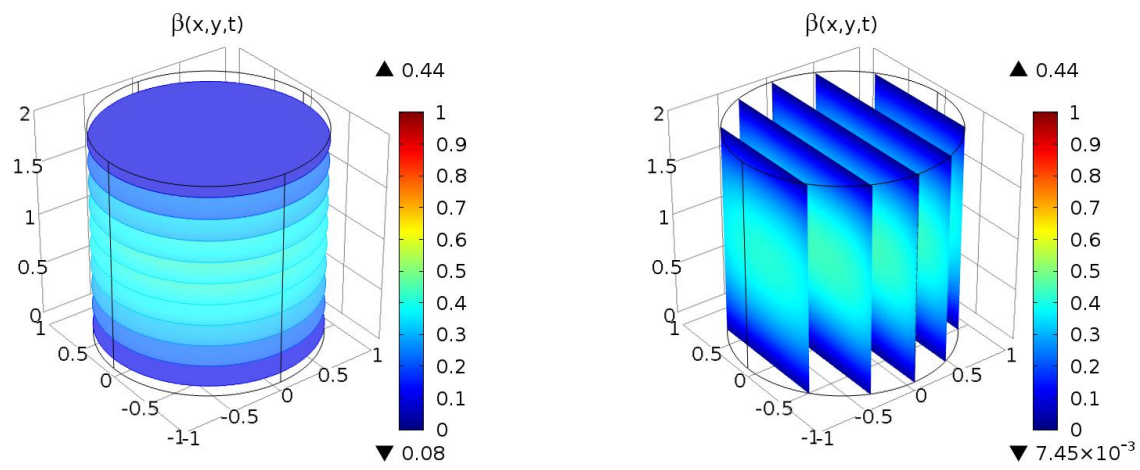

Figure 20: Optimal sterile mosquito release $\beta^{*}$ for $\varepsilon=10$ with bounds $0.01 \leq \alpha(t) \leq 0.09,0 \leq \beta(t) \leq 1$, initial conditions $w_{0}=5, s_{0}=5$. The horizontal plane is the $x y$-plane on the unit circle and the vertical is the $t$-direction for times $t=[0,5]$. 